ARTICLE

https://doi.org/10.1038/s41467-019-11270-1

\title{
Adipose group 1 innate lymphoid cells promote adipose tissue fibrosis and diabetes in obesity
}

Hongdong Wang ${ }^{1,6}$, Lei Shen ${ }^{2,6}$, Xitai Sun (10 ${ }^{3,6}$, Fangcen Liu ${ }^{4,6}$, Wenhuan Feng ${ }^{1}$, Chunping Jiang ${ }^{3}$, Xuehui Chu ${ }^{3}$, Xiao $\mathrm{Ye}^{4}$, Can Jiang ${ }^{1}$, Yan Wang ${ }^{1}$, Pengzi Zhang ${ }^{1}$, Mengwei Zang ${ }^{5}$, Dalong Zhu ${ }^{1,6} \&$ Yan $\mathrm{Bi}^{1}$

Pathogenic factors driving obesity to type 2 diabetes (T2D) are not fully understood. Group 1 innate lymphoid cells (ILC1s) are effectors of innate immunity and enriched in inflamed tissues. Here we show that the number of adipose ILC1s increases in obese T2D patients and correlates with glycemic parameters and with the number of ILC1s in the blood; circulating ILC1 numbers decrease as a result of metabolic improvements after bariatric surgery. In vitro co-culture experiments show that human adipose ILC1s promote adipose fibrogenesis and $\mathrm{CD}_{11 \mathrm{c}^{+}}$macrophage activation. Reconstruction of the adipose ILC1 population in Prkdc ${ }^{-/-} / \mathrm{L}^{2} \mathrm{rg}^{-/-}$mice by adoptive transfer drives adipose fibrogenesis through activation of TGF $\beta 1$ signaling; however, transfer of Ifng ${ }^{-/-}$ILC1s has no effect on adipose fibrogenesis. Furthermore, inhibiting adipose accumulation of ILC1s using IL-12 neutralizing antibodies attenuates adipose tissue fibrosis and improves glycemic tolerance. Our data present insights into the mechanisms of local immune disturbances in obesity-related T2D.

\footnotetext{
${ }^{1}$ Department of Endocrinology, Drum Tower Hospital Affiliated to Nanjing University Medical School, Nanjing 210008, China. ${ }^{2}$ Shanghai Institute of Immunology, Shanghai Jiao Tong University School of Medicine, Shanghai 200025, China. ${ }^{3}$ Department of General Surgery, Drum Tower Hospital Affiliated to Nanjing University Medical School, Nanjing 210008, China. ${ }^{4}$ Drum Tower Hospital Clinical College of Nanjing Medical University, Nanjing 210008, China. ${ }^{5}$ Department of Molecular Medicine, Barshop Institute for Longevity and Aging Studies, University of Texas Health San Antonio, San Antonio, TX $78229-$ 3900, USA. ${ }^{6}$ These authors contributed equally: Hongdong Wang, Lei Shen, Xitai Sun, Fangcen Liu, Dalong Zhu. Correspondence and requests for materials should be addressed to Y.B. (email: biyan@nju.edu.cn)
} 
T ype 2 diabetes (T2D), one of the largest public health challenges worldwide, is related to the dramatically increased incidence of obesity ${ }^{1,2}$, with $\sim 60-90 \%$ of patients with T2D being obese ${ }^{3,4}$. Emerging findings indicate that innate and adaptive immune responses in adipose tissue have critical functions in the regulation of metabolic homeostasis, while in obesity, type 1 inflammation-associated immune cells are predominant in adipose tissue and exert metabolically deleterious impacts ${ }^{5}$. Clarifying the precise alteration of the adipose tissue immune system and subsequent effect on metabolic homeostasis is urgently needed to counter the rise in obesity-associated T2D.

Innate lymphoid cells (ILCs) are effectors and regulators of innate immunity, and can be found in both lymphoid and nonlymphoid peripheral tissues ${ }^{6}$. On the basis of the expression of transcription factors, cell surface markers, and effector cytokines, mature ILCs can be classified into groups 1, 2, or $3 \mathrm{ILCs}^{6}$, of which, group 1 ILCs include conventional natural killer (cNK) cells and subsets of unconventional NK cells ${ }^{7}$. During the development of group 1 ILCs, key transcription factors TOX, NFIL3, and Id 2 are required at an early stage of differentiation, whereas the master transcription factor, T-bet, is important for the interferon (IFN)- $\gamma$-producing function ${ }^{6,8}$. Interestingly, ILC1s were previously reported to be particularly prominent under inflammatory conditions in mucosal tissues from Crohn's disease patients 9,10 , indicating the role for ILC1s in the pathogenesis of gut mucosal inflammation. A study has shown that accumulation of adipose ILC1s directly induces local inflammation and systemic insulin resistance in high-fat-diet (HFD)-fed mice ${ }^{11}$. However, whether adipose ILC1s contributes to the development of T2D in obese individuals is not clear.

Adipose tissue fibrosis in obese individuals, caused by pathologically excessive accumulation of the extracellular matrix (ECM), is a hallmark of malfunction that is linked to insulin resistance and $\mathrm{T} 2 \mathrm{D}^{12-14}$, while repression of adipose tissue fibrosis improves glucose homeostasis ${ }^{15}$. Adipose tissue macrophages (ATMs) are reported to have an important function in regulating adipose tissue fibrosis: macrophage-secreted factors are shown to promote a profibrotic phenotype in preadipocytes; ${ }^{16}$ in addition, proinflammatory $\mathrm{CD}_{11 \mathrm{c}^{+}}$macrophages drive adipose tissue fibrogenesis by regulating distinct cell types ${ }^{17,18}$. Adipose ILC1s were reported to promote $\mathrm{CD}_{11 \mathrm{c}^{+}}$macrophage activation in HFD- induced mice ${ }^{11}$, but whether adipose ILC1s regulate adipose tissue fibrogenesis and the underlying mechanisms are unclear.

Here, we show that the number of adipose ILC1s increases in obese T2D patients and induces adipose fibrogenesis in an IFN$\gamma$-dependent fashion. Reconstruction of adipose ILC1s in Prkdc ${ }^{-/} I L 2 \mathrm{rg}^{-/-}$mice drives adipose fibrogenesis through activation of transforming growth factor $\beta-1$ (TGF- $\beta 1$ ) signaling, whereas inhibiting adipose ILC1s accumulation attenuates adipose tissue fibrosis and improves glycemic intolerance. Thus, our data present mechanistic insights into local immune disturbances in obesity-associated T2D.

\section{Results}

Adipose ILC1s correlate with the development of obesityassociated T2D. To evaluate the role of adipose ILC1s in the development of obese T2D, we enrolled control subjects $(n=36)$, obese individuals $(n=27)$, and obese T2D patients $(n=22)$, whose clinical and biochemical characteristics of the enrolled subjects are summarized in Table 1. There were no differences in age and sex between controls, obese subjects, and obese T2D patients.

Circulating and adipose ILC1s were identified as $\mathrm{Lin}^{-} \mathrm{CD} 45^{+}$ $\mathrm{CD}^{2} 7^{+} \mathrm{CD} 117^{-} \mathrm{CRTH} 2^{-} \mathrm{NKP} 44^{-}$lymphocytes (Fig. 1a), with isotype control data shown in Supplementary Fig. 1a. Compared with the controls, the absolute numbers of circulating ILC1s (cells $\mathrm{ml}^{-1}$ ) were significantly increased in obese subjects, which were further higher in obese T2D patients (Fig. 1b). Clinical characteristics of subjects with adipose tissue samples analyzed are summarized in Supplementary Table 1. Significantly higher numbers of ILC1s resident in the omental adipose tissue (cells $\mathrm{mg}^{-1}$ ) were detected in the obese group compared with the control group ( $15 \pm 3$ vs. $5 \pm 3, P<0.001$, two-way ANOVA tests followed by Bonferroni post hoc test, Fig. 1c), which was even higher in obese T2D patients (obese T2D vs. obese: $23 \pm 3$ vs. $15 \pm 3, P<0.001$, twoway ANOVA tests followed by Bonferroni post hoc test, Fig. 1c).

Meaningfully, a positive correlation between the number of circulating and adipose ILC1s was observed $(r=0.838, P=0.000$, Spearman's correlation, $t$ test, Fig. 1d). After controlling for the age and sex, circulating ILC1s were positively associated with fasting glucose levels $(r=0.713, P=0.000$, Spearman's partial correlation, $t$ test), postprandial blood glucose levels $(r=0.756$,

\begin{tabular}{|c|c|c|c|c|c|c|c|}
\hline & \multirow[t]{2}{*}{ Control } & \multirow[t]{2}{*}{ Obese } & \multirow[t]{2}{*}{ Obese T2D } & \multicolumn{4}{|l|}{ P-values } \\
\hline & & & & Overall & $\begin{array}{l}\text { Control } \\
\text { vs. obese }\end{array}$ & $\begin{array}{l}\text { Control vs. } \\
\text { obese T2D }\end{array}$ & $\begin{array}{l}\text { Obese vs. } \\
\text { obese T2D }\end{array}$ \\
\hline N & 36 & 27 & 22 & - & - & - & - \\
\hline Age (years) & $42.6 \pm 6.5$ & $40.0 \pm 13.6$ & $38.7 \pm 11.2$ & 0.353 & - & - & - \\
\hline Male, $n(\%)$ & $8(22.2)$ & $8(29.6)$ & $6(27.3)$ & 0.796 & - & - & - \\
\hline Fasting glucose $\left(\mathrm{mmol} \mathrm{I}^{-1}\right)$ & $4.8 \pm 0.4$ & $5.4 \pm 0.9$ & $11.0 \pm 3.5$ & 0.000 & 0.189 & 0.000 & 0.000 \\
\hline $2 \mathrm{~h}$ post $\left(\mathrm{mmol} \mathrm{I}^{-1}\right)^{\mathrm{a}}$ & $5.7 \pm 0.9^{a}$ & $7.1 \pm 1.5$ & $16.7 \pm 4.1$ & 0.000 & 0.309 & 0.000 & 0.000 \\
\hline Fasting insulin $\left(\mathrm{mlU} \mathrm{ml}{ }^{-1}\right)$ & $7.0 \pm 8.6$ & $22.5 \pm 14.2$ & $31.5 \pm 19.9$ & 0.000 & 0.000 & 0.000 & 0.027 \\
\hline HOMA-IR (units) & $1.5 \pm 2.0$ & $5.7 \pm 4.0$ & $14.8 \pm 9.1$ & 0.000 & 0.003 & 0.000 & 0.000 \\
\hline Triglycerides $\left(\mathrm{mmol} \mathrm{I}^{-1}\right)$ & $1.2 \pm 0.5$ & $1.8 \pm 0.8$ & $4.2 \pm 3.5$ & 0.000 & 0.017 & 0.000 & 0.000 \\
\hline Total cholesterol (mmol I-1) & $4.6 \pm 0.9$ & $4.5 \pm 0.9$ & $5.1 \pm 1.1$ & 0.071 & - & - & - \\
\hline
\end{tabular}



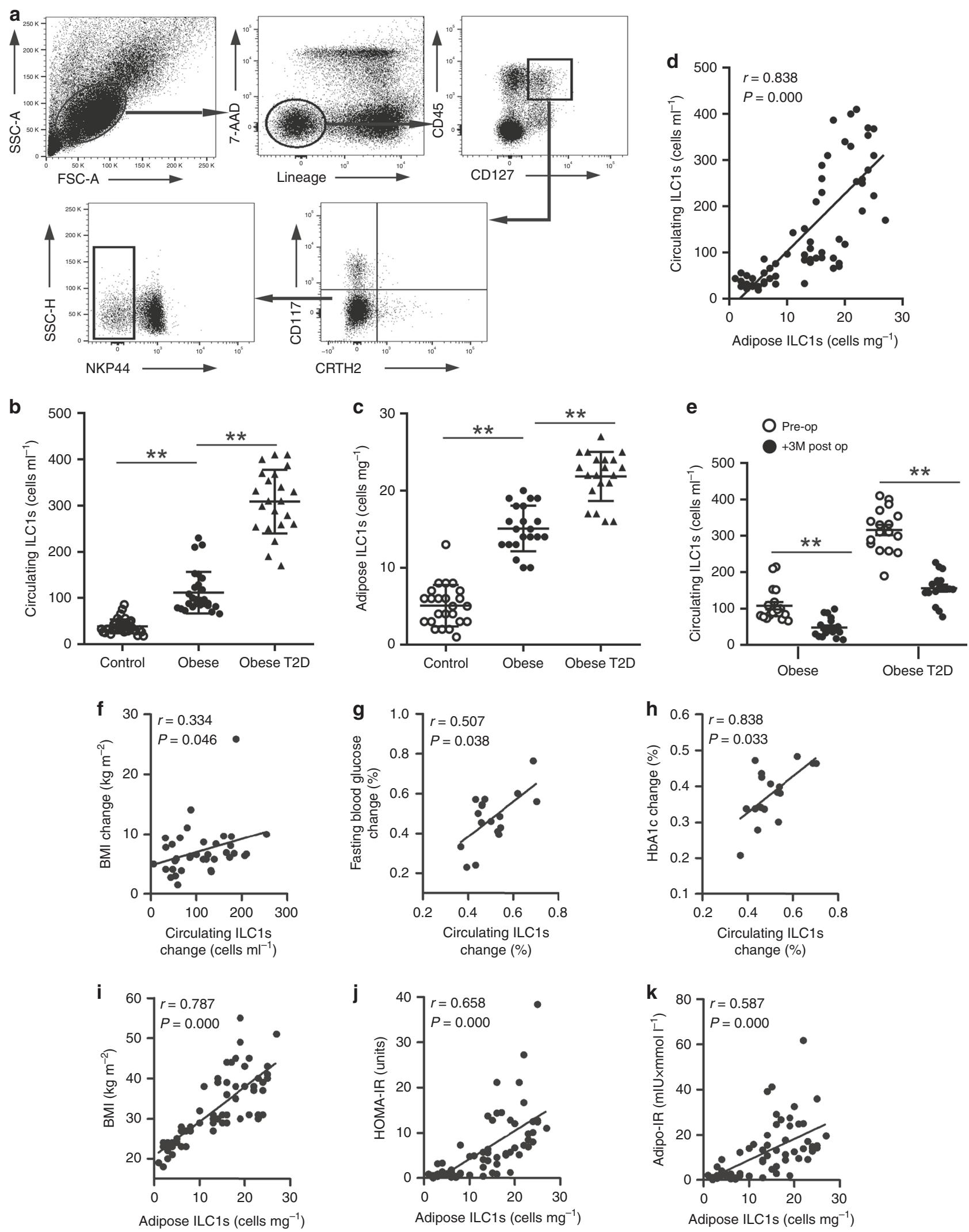

$P=0.000$, Spearman's partial correlation, $t$ test), and HbA1c levels ( $\mathrm{r}=0.801, P=0.000$, Spearman's partial correlation, $t$ test). Furthermore, the numbers of adipose ILC1s were also positively related with fasting glucose levels $(r=0.677, P=0.000$, Spearman's partial correlation, $t$ test), postprandial blood glucose levels
( $\mathrm{r}=0.701, P=0.000$, Spearman's partial correlation, $t$ test), and HbA1c levels $(r=0.753, P=0.000$, Spearman's partial correlation, $t$ test), after adjusted for age and sex (Table 2). In 19 obese subjects and 17 obese T2D patients with 3 months of follow-up (Supplementary Table 2), compared with their baseline levels, the 
Fig. 1 Adipose ILC1s correlate with the development of obesity and obese T2D. a Analysis of ILC1s by flow cytometry. $\mathbf{b}$ Absolute numbers (cells ml ${ }^{-1}$ ) of circulating ILC1s in control subjects $(n=36)$, obese subjects $(n=27)$, and obese T2D patients $(n=22)$. c Absolute numbers (cells mg $\left.{ }^{-1}\right)$ of adipose ILC1s in controls $(n=24)$, obese subjects $(n=21)$, and obese T2D patients $(n=20)$. $\mathbf{d}$ Correlation between adipose ILC1s numbers and circulating ILC1s $(n=$ 65). e Numbers of circulating ILC1s before and at 3 months after bariatric surgery. $\mathbf{f}$ Correlation between reduction of circulating ILC1s and decrement of BMI levels in obese patients with 3-months follow-up $(n=36) . \mathbf{g}$, $\mathbf{h}$ Correlations between decrement of fasting glucose levels and HbA1c levels with reduction of circulating ILC1s in obese T2D subgroup $(n=17)$. i-k Correlations between BMI, HOMA-IR, and Adipo-IR with adipose ILC1s $(n=65)$. ${ }^{\star \star} P<$ 0.01 (two-way ANOVA tests followed by Bonferroni post hoc test). Error bars indicate s.d. ILC innate lymphoid cells

numbers of circulating ILC1s were all significantly reduced after 3 months post surgery (Fig. 1e). Importantly, in all obese subjects with 3 months of follow-up, the reduction of circulating ILC1s correlated with the decrement of body mass index (BMI) levels ( $\mathrm{r}$ $=0.334, P=0.046$, Spearman's correlation, $t$ test, Fig. 1f). Moreover, in obese T2D subgroup, the reduction of circulating ILC1s positively correlated with decrement of fasting glucose levels ( $\mathrm{r}=0.507, P=0.038$, Spearman's correlation, $t$ test, Fig. $1 \mathrm{~g}$ ) and HbA1c levels $(r=0.838, P=0.033$, Spearman's correlation, $t$ test, Fig. 1h).

Relationship between adipose ILC1s and insulin resistance was further evaluated. As shown in Fig. 1i-k, adipose ILC1s positively correlated with BMI $(\mathrm{r}=0.787, P=0.000$, Spearman's correlation, $t$ test), homeostasis model assessment of insulin-resistance (HOMA-IR) values $(\mathrm{r}=0.658, P=0.000$, Spearman's correlation, $t$ test), and adipose tissue insulin-resistance index (Adipo-IR) ( $\mathrm{r}=0.587, P=0.000$, Spearman's correlation, $t$ test $)$.

Adipose ILC1s promote adipose fibrogenesis in humans. We next evaluated the potential role of adipose ILC1s in the development of adipose tissue fibrosis. Compared with the control subjects, obese individuals showed more collagen fibers around adipocytes in the adipose tissue (Supplementary Fig. 1b), which was further confirmed by a higher percentage of fibrotic area and higher expression levels of fibrotic-related genes (Supplementary Fig. 1c). The percentage of positively stained area indicated for adipose tissue fibrosis correlated with the number of adipose ILC1s ( $\mathrm{r}=0.851, P=0.000$, Spearman's correlation, $t$ test), BMI ( $\mathrm{r}=0.785, P=0.000$, Spearman's correlation, $t$ test), HOMA-IR ( $\mathrm{r}=0.714, P=0.000$, Spearman's correlation, $t$ test), and AdipoIR ( $\mathrm{r}=0.658, P=0.000$, Spearman's correlation, $t$ test) (Fig. 2a; Supplementary Table 3 ). Multivariate stepwise regression analysis further revealed that the number of adipose ILC1s was the major determinant of the variations of adipose fibrosis level $(\beta=0.689$, $P=0.000)$ (adjusted $\mathrm{R}^{2}$ for the model $=0.728$ ) (Table 3 ).

Notably, compared with the controls, higher expressions of IFN- $\gamma$ were found in adipose ILC1s from obese subjects, while adipose ILC1s from obese subjects and obese T2D patients expressed similar levels of IFN- $\gamma$ (Fig. 2b). To address the role of adipose ILC1s in adipose tissue fibrogenesis, stromal vascular fractions (SVFs) from control subjects were co-cultured with either $\mathrm{CD}_{4} 5^{+}$cells or ILCs depleted CD45 ${ }^{+}$cells sorted from the SVFs of obese T2D patients (Fig. 2c; Supplementary Fig. 1d); meanwhile, both trehalose-6,6'-dimycolate, a mycobacterial cell wall glycolipid that is known to be a macrophage-inducible c-type lectin (Mincle) ligand ${ }^{17}$, and palmitate were added to mimic the in vivo microenvironment. Three days after co-culture, higher numbers of $\mathrm{CD}_{11 \mathrm{c}^{+}}$macrophages (Fig. 2d; Supplementary Fig. 1e) and significantly increased mRNA expression of Mincle, inducible nitric oxide synthase (iNOS), and transforming growth factor beta-1 ( $T g f b 1)$ were observed in SVFs of the ILCs nondepleted group relative to those in the ILCs depleted group (Fig. 2e). Moreover, mRNA expression of key collagen genes, Colla and Col3a, was notably increased in SVFs of the ILCs nondepleted group (Fig. 2e).

\begin{tabular}{|c|c|c|c|c|}
\hline & \multicolumn{2}{|c|}{$\begin{array}{l}\text { Circulating ILC1s } \\
(\mathbf{N}=\mathbf{8 5})\end{array}$} & \multicolumn{2}{|c|}{$\begin{array}{l}\text { Adipose ILC1s } \\
(N=65)\end{array}$} \\
\hline & $\mathbf{R}$ & $P$ & $\mathbf{r}$ & $P$ \\
\hline$\overline{\text { FBG }\left(\mathrm{mmol} \mathrm{I} \mathrm{I}^{-1}\right)}$ & 0.715 & 0.000 & 0.682 & 0.000 \\
\hline 2 -h post $\left(\mathrm{mmol} \mathrm{I}^{-1}\right)$ & $0.750^{a}$ & 0.000 & $0.706^{b}$ & 0.000 \\
\hline $\mathrm{HbA1c}(\%)$ & 0.790 & 0.000 & 0.755 & 0.000 \\
\hline \multicolumn{5}{|c|}{ After adjusted for age and sex } \\
\hline $\mathrm{FBG}(\mathrm{mmol} \mathrm{I}-1)$ & 0.713 & 0.000 & 0.677 & 0.000 \\
\hline 2 -h post $\left(\mathrm{mmol} \mathrm{I}^{-1}\right)$ & $0.756^{a}$ & 0.000 & $0.701^{b}$ & 0.000 \\
\hline HbA1c (\%) & 0.801 & 0.000 & 0.753 & 0.000 \\
\hline
\end{tabular}

To further confirm whether adipose ILC1s could promote adipose tissue fibrogenesis independent of other obesity-induced CD $45^{+}$cells, adipose ILCs were co-cultured with SVFs of control subjects (Fig. 2f). Three days after co-culture, expressions of fibrosis-related genes and ECM regulators, including Mincle, $i N O S, T g f b 1$, Colla, and Col3a, were significantly increased in SVFs co-cultured with ILCs compared with those in the control group, while these changes were notably reversed by administration of neutralizing IFN- $\gamma$ antibody (Fig. $2 \mathrm{~g}$ ). These findings indicate that the effect of adipose ILC1s on adipose tissue fibrogenesis is independent of other obesity-induced CD $45^{+}$cells and is potentially mediated by IFN- $\gamma$.

Reconstruction of the adipose ILC1 population induces adipose fibrosis. To further determine the causative role of adipose ILC1s in adipose tissue fibrosis, we adoptively transferred purified adipose ILC1s from HFD-fed wild-type mice into Prkdc ${ }^{-/} I \mathrm{~L}_{2 g^{-/}}$ mice and fed HFD for 4 weeks (Fig. 3a). Adipose ILC1s in visceral adipose tissue (VAT) were identified as $\mathrm{Lin}^{-} \mathrm{NK} 1.1^{+} \mathrm{NKP} 46^{+} \mathrm{T}-$ bet $^{+}$ Eomes ${ }^{-}$DX5 ${ }^{-}$lymphocytes (Supplementary Fig. 1f), as reported recently ${ }^{11}$. Four weeks after cell transfer and HFD feeding, we observed that adipose ILC1s significantly accumulated in recipient VAT of mice receiving adipose ILC1 transfer compared with untreated control mice fed with normal diet (CHOW) or HFD (Fig. 3b). There was no appreciable difference in body weight between mice receiving ILC1 transfer and HFD mice receiving PBS injection (Fig. 3c), whereas glucose intolerance tended to be more severe in mice receiving ILC1 transfer (Fig. 3d). Expression of key collagen genes, Colla and Col3a, was significantly higher in adipose tissue of mice receiving ILC1 transfer (Fig. 3e). In addition, expression of platelet derived growth factor subunit $\mathrm{b}(P d g f b)$ and alpha-smooth muscle actin (Acta2), which regulates fibroblast proliferation and encodes the myofibroblast marker $\alpha$-smooth muscle actin $(\alpha S M A)$, respectively, was significantly increased in mice receiving ILC1 transfer (Fig. 3e). Masson's trichrome and Sirius red staining further revealed extensive interstitial fibrosis in VAT of mice 
a

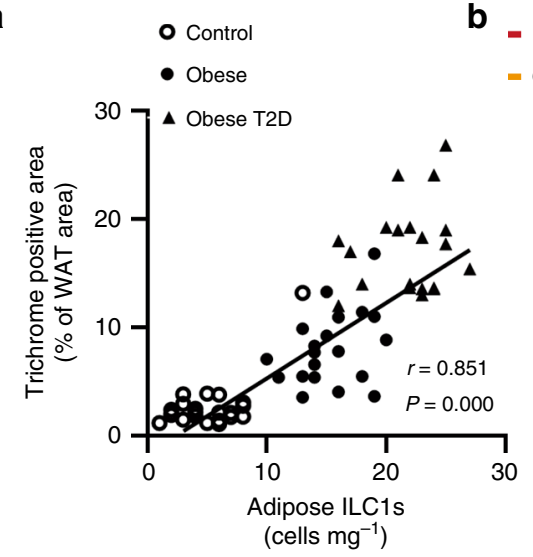

d

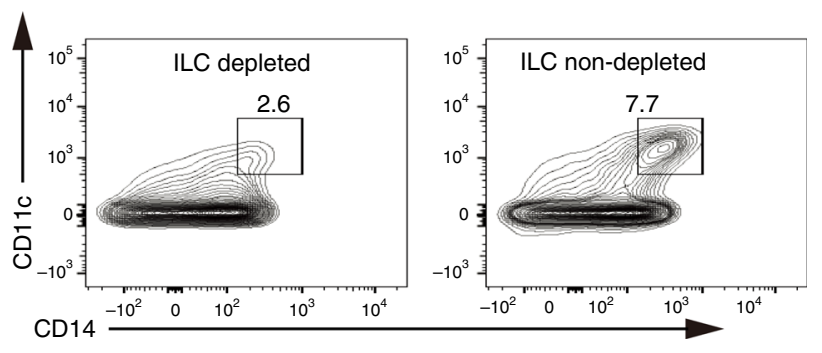

$\mathbf{f}$
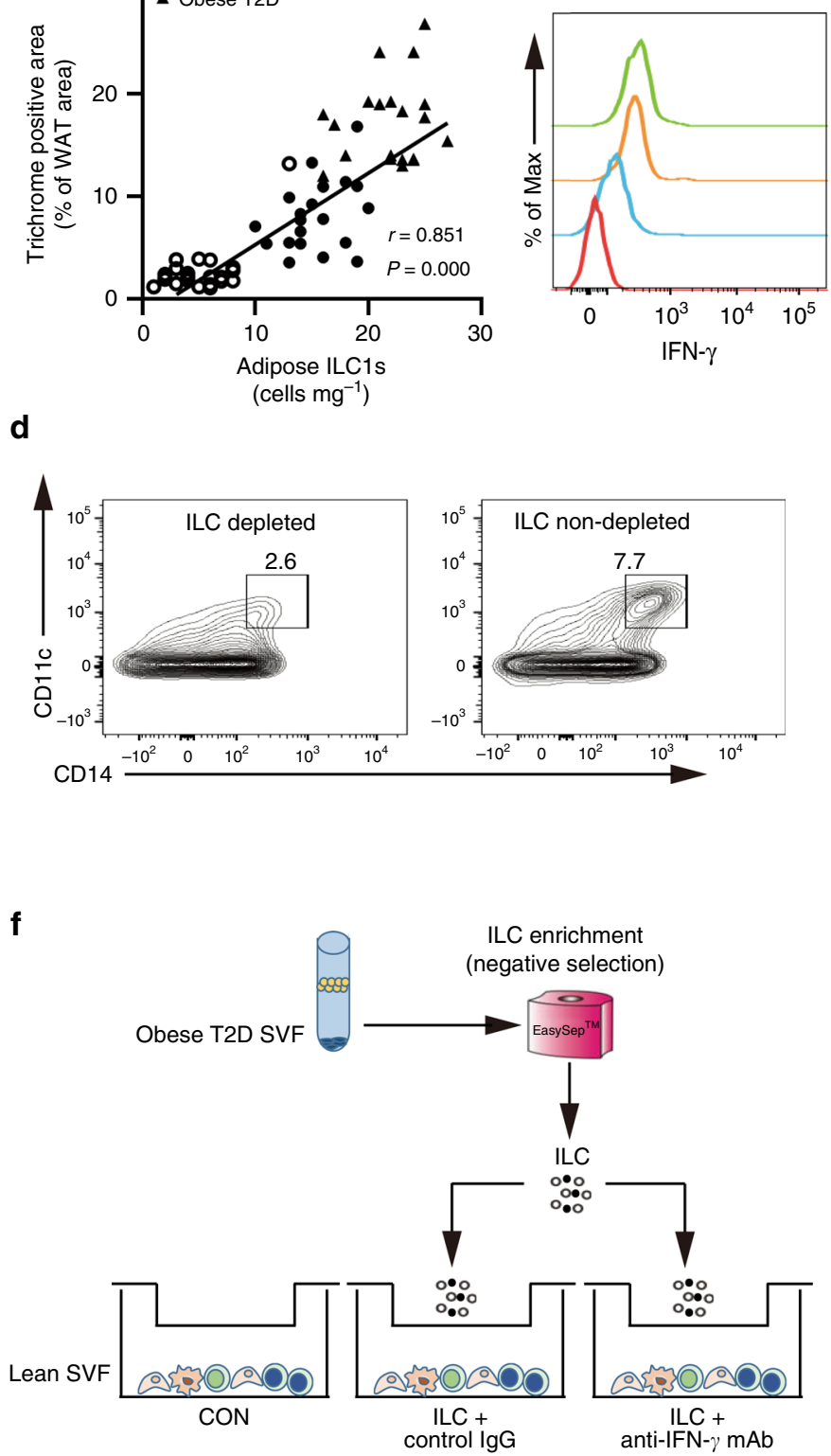

C
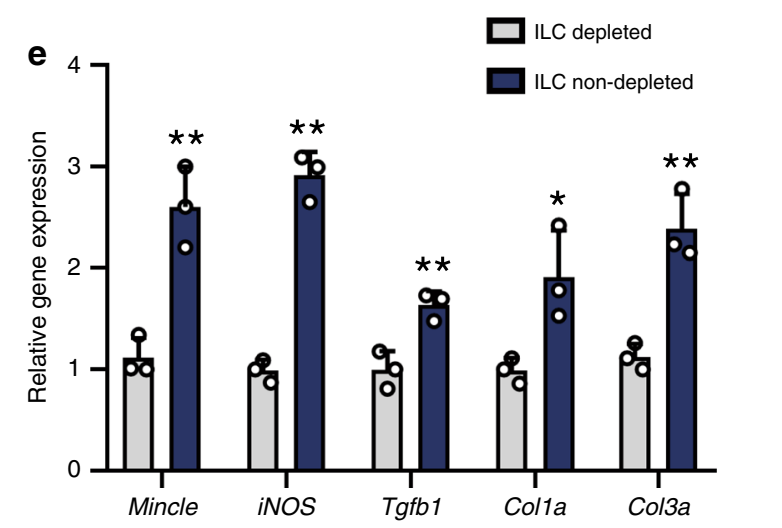

g

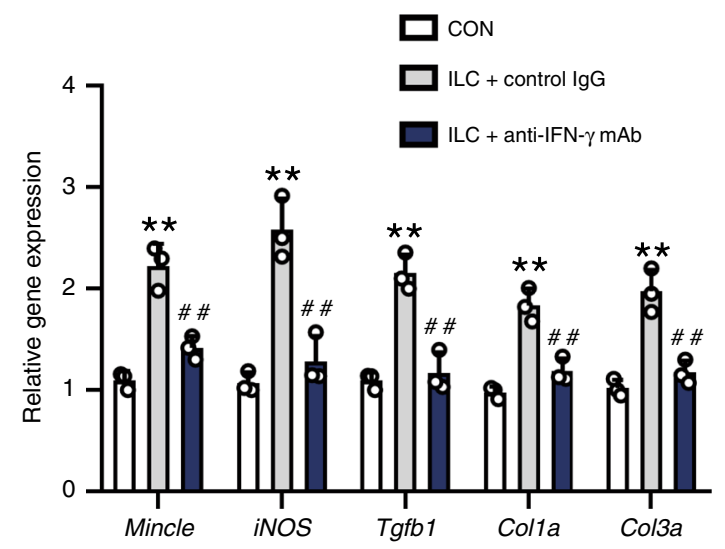

Fig. 2 Adipose ILC1s contribute to adipose tissue fibrogenesis in humans. a Association of adipose ILC1s with trichrome C staining area. b Representative histogram of IFN- $\gamma$ in adipose ILC1s from control subjects, obese subjects, and obese T2D patients. The data are representative of three independent experiments. c-e SVFs isolated from control subjects were co-cultured with either CD45+ cells or ILCs-depleted CD45 ${ }^{+}$cells sorted from the SVFs of obese T2D patients; meanwhile, trehalose-6, $6^{\prime}$-dimycolate $\left(5 \mu \mathrm{g}\right.$ well $\left.{ }^{-1}\right)$ and palmitate $(200 \mu \mathrm{M})$ were added in the lower chamber. Three days after coculture, SVFs in the lower chamber were collected for further detection. $\mathbf{c}$ Illustration of the co-culture experiments. $\mathbf{d}$ Representative plots of CD11c ${ }^{+}$ macrophages in SVFs of the lower chamber. e mRNA expression of Mincle, iNOS, and fibrosis-related genes (Tgfb1, Colla, Col3a) in SVFs of the lower chamber. ${ }^{*} P<0.05$ vs. ILC depleted group; ${ }^{* *} P<0.01$ vs. ILC depleted group (unpaired two-way Student's $t$ test). The data are representative of three independent experiments. $\mathbf{f}, \mathbf{g}$ In another set of the co-culture experiment, $1 \times 10^{8}$ cells of the SVF from obese T2D patients were magnetically enriched for ILCs using negative immunomagnetic selection. Then, adipose ILCs were co-cultured with SVFs of control subjects $\left(1 \times 10^{6}\right.$ well-1). Trehalose-6, $6^{\prime}-$ dimycolate $\left(5 \mu \mathrm{g} \mathrm{well}^{-1}\right)$, palmitate $(200 \mu \mathrm{M})$, recombinant human IL-12 $\left(20 \mathrm{ng} \mathrm{ml}^{-1}\right)$, and recombinant human IL-18 (20 ng ml $\left.{ }^{-1}\right)$ were supplemented in the upper chamber, with neutralizing IFN- $\gamma$ antibody $\left(20 \mathrm{n} \mathrm{ml}^{-1}\right)$ or IgG isotype control antibody (20 $\mathrm{ng} \mathrm{ml}{ }^{-1}$ ) added in separate group. SVFs isolated from adipose tissue of control subjects were cultured alone and determined as control group. After co-culture for $72 \mathrm{~h}$, SVFs in the lower chamber were collected for further detection. $\mathbf{f}$ Graphical illustration of the co-culture experiments. $\mathbf{g}$ mRNA expression of Mincle, iNOS, Tgfb1, Col1a, and Col3a in SVFs of the lower chamber. ${ }^{\star \star} P<0.01$ vs. control group; ${ }^{\#} P<0.01 \mathrm{vs}$. ILC +Control IgG group (two-way ANOVA tests followed by Bonferroni post hoc test). The data are representative of three independent experiments. Error bars indicate s.d. IFN interferon, ILC innate lymphoid cells 
Table 3 Multivariate stepwise regression analysis

\begin{tabular}{|c|c|c|c|c|}
\hline & B & Standard error & Standard $\beta$ & $P$-value \\
\hline \multicolumn{5}{|c|}{ Positive stained area } \\
\hline Adipose ILC1s & 0.689 & 0.130 & 0.779 & 0.000 \\
\hline $\mathrm{BMI}$ & -0.087 & 0.103 & -0.106 & 0.403 \\
\hline Adipo-IR & -0.152 & -0.104 & -0.221 & 0.149 \\
\hline HOMA-IR & 0.555 & 0.269 & 0.373 & 0.044 \\
\hline
\end{tabular}

receiving ILC1 transfer relative to HFD mice receiving PBS injection (Fig. 3f). In agreement, there was a significant increase in the area of aSMA-positive cells in mice receiving ILC1 transfer relative to HFD mice receiving PBS injection, as assessed by immunohistological analyses (Fig. $3 \mathrm{~g}, \mathrm{~h}$ ). In addition, the hepatic triglyceride content and serum-free fatty acid concentrations tended to increase in mice receiving ILC1 transfer than in HFD mice receiving PBS injection, which did not reach statistical significance (Supplementary Fig. 1g, h).

Adipose ILC1s regulate ATMs and TGF- $\beta 1$ signaling. The appearance of crown-like structures (CLS) mainly composed of clusters of $\mathrm{CD}_{11 \mathrm{c}^{+}}$macrophages is associated with adipose tissue fibrosis ${ }^{17}$. Following HFD feeding, CLS was visualized in adipose tissue by IHC with staining for F4/80, while we found that mice receiving adipose ILC1 transfer and HFD feeding had substantially more CLS than did HFD mice receiving PBS injection (Fig. 4a). Accordingly, we detected higher numbers of CD11c ${ }^{+}$and $\mathrm{CD} 11 \mathrm{c}^{+}$ $\mathrm{CD}^{206^{+}}$macrophages in mice receiving ILC1 transfer compared with HFD mice receiving PBS injection (Fig. 4b; Supplementary Fig. 2a, b), in line with increased phosphorylation levels of STAT1 (Fig. 4c), which is associated with proinflammatory $\mathrm{CD}_{11 c^{+}}$ macrophage activation ${ }^{19}$. Interestingly, immunofluorescence of pSTAT1 and the macrophage marker F4/80 showed pSTAT1 staining mostly colocalized with F4/80, which indicates that pSTAT1 is specifically expressed in ATMs (Fig. 4d). In vitro co-culture experiments using adipose ILC1s and bone marrowderived macrophages (BMDMs) showed that, expressions of proinflammatory macrophage markers, IL-6, NOS2, and TNF- $\alpha$ were significantly increased in BMDMs co-cultured with isotype IgG treated adipose ILC1s compared with the control group, whereas these changes were notably reversed after administration of neutralizing IFN- $\gamma$ antibody (Supplementary Fig. 2c).

Notably, TGF- $\beta 1$ mRNA expression was markedly higher in VAT of mice receiving ILC1 transfer than that in HFD mice receiving $\mathrm{PBS}$ injection (Fig. 4e). Intriguingly, immunofluorescence showed that TGF- $\beta 1$ staining was mostly colocalized with F4/80 (Fig. 4f). Moreover, TGF- $\beta 1$ activity, as determined by phosphorylation of Smad3, was enhanced after ILC1 transfer (Fig. 4g). In agreement with elevated iNOS expression in human co-culture experiment, upregulation of $i N O S$ expression was also observed in VAT of mice receiving ILC1 transfer; in contrast, Mincle mRNA expression tended to be higher in mice receiving ILC1 transfer, but did not reach significance (Fig. 4h).

Adipose ILC1s induce adipose fibrosis via IFN- $\gamma$ production. To validate that adipose ILC1s-derived IFN- $\gamma$ was responsible for adipose tissue fibrogenesis and glycemic intolerance in obesity, we adoptively transferred adipose ILC1s from HFD-fed Ifng ${ }^{-/-}$mice or wild-type mice into Prkdc ${ }^{-/} I L 2 \mathrm{rg}^{-/-}$mice and fed HFD for 6 weeks (Fig. 5a). Although no significant difference in body weight was found (Fig. 5b), mice receiving ILC1 transfer showed higher fasting glucose level and more severe glycemic intolerance compared with control mice fed with normal diet or HFD mice receiving PBS injection (Fig. $5 \mathrm{c}, \mathrm{d}$ ). However, following Ifng ${ }^{-/-}$ ILC1 transfer and HFD feeding in Prkdc ${ }^{-/} I \mathrm{~L}_{2} \mathrm{rg}^{-/-}$mice, no significant difference in fasting glucose level or aggravating glycemic intolerance was found compared with HFD mice receiving PBS injection (Fig. 5c, d). Furthermore, expression of fibrosisrelated genes and ECM regulators, including Colla, Col3a, Acta2, $T g f b 1, P d g f b$, and $i N O S$ in VAT, was significantly increased in mice receiving ILC1 transfer compared to HFD mice receiving PBS injection and mice receiving Ifng ${ }^{-/-}$ILC1 transfer (Fig. 5e). Masson's trichrome and Sirius red staining further showed extensive interstitial fibrosis in VAT of mice receiving ILC1 transfer relative to HFD mice receiving PBS injection and mice receiving Ifng ${ }^{-/-}$ILC1 transfer (Fig. 5f). These findings demonstrate that IFN- $\gamma$ production mediates the effects of adipose ILC1s on adipose tissue fibrogenesis and glycemic disturbance.

Inhibiting adipose ILC1 accumulation attenuates fibrosis. IL12 signaling is required for the proliferation of adipose ILC1s during diet-induced obesity ${ }^{11}$. To investigate the therapeutic potential of targeting adipose ILC1s to improve adipose tissue fibrosis and glycemic intolerance in obesity, IL-12 neutralizing antibody was utilized to block IL-12 signaling activation and subsequent adipose ILC1s accumulation in HFD-fed Rag1-/mice. Flow-cytometric analyses showed that adipose ILC1s in VAT were 2.5-fold higher after HFD feeding than in normal chow fed mice, whereas anti-IL-12 mAb treatment in HFD-fed mice significantly decreased numbers of adipose ILC1s (Fig. 6a, b). Consistently, $\mathrm{CD} 11 \mathrm{c}^{+}$and $\mathrm{CD} 11 \mathrm{c}^{+} \mathrm{CD} 206^{+}$macrophages in VAT were also reduced after anti-IL-12 mAb administration; in contrast, $\mathrm{CD}^{206^{+}}$macrophages were unchanged in anti-IL-12 mAb-treated HFD-fed mice compared with control IgG treatment (Fig. 6c; Supplementary Fig. 2d). Although body weight and fasting blood glucose were not affected (Fig. 6d, e), improved glucose intolerance was observed in anti-IL-12 mAb-treated mice, compared with mice treated with control IgG (Fig. 6f). Strikingly, abnormal collagen deposition was notably reduced in VAT of anti-IL-12 mAb-treated mice, as shown by Masson's and Sirius Red staining (Fig. 6g). Meanwhile, expression of fibrosis-related genes and ECM regulators, including Col1a, Col3a, Acta2, Tgfb1, and Pdgfb, was significantly decreased after anti-IL-12 mAb treatment (Fig. 6h). The beneficial effect of anti-IL-12 mAb treatment on interstitial fibrosis was also evidenced by reduced gene expression of iNOS, in accordance with decreased $\mathrm{CD}_{11 \mathrm{c}^{+}}$macrophages (Fig. 6h). In addition, histological examination revealed that hepatic steatosis was markedly attenuated in anti-IL-12 mAb-treated mice relative to mice treated with control IgG (Fig. 6i). Consistently, hepatic triglyceride content and serum-free fatty acid concentrations were significantly reduced in anti-IL-12 mAb-treated mice compared with mice treated with control IgG (Fig. 6j, k).

To further validate the potential of targeting adipose ILC1s to improve adipose tissue fibrogenesis and glycemic intolerance in obesity, IL-12 neutralizing antibody was administrated in HFDfed wild-type mice. Compared with mice treated with control IgG, improved glucose intolerance was observed in anti-IL-12 mAb-treated wild-type mice (Supplementary Fig. 2e). Moreover, anti-IL-12 mAb treatment significantly decreased expression of fibrosis-related genes and ECM regulators including Colla, Col3a, Acta2, Tgfbl, Pdgfb, and $i N O S$ in VAT of anti-IL-12 mAb-treated wild-type mice (Supplementary Fig. 2f). In addition, Masson's trichrome and Sirius red staining revealed abnormal collagen deposition in VAT of HFD-fed mice treated with control IgG, whereas anti-IL-12 mAb-treated wild-type mice showed significantly less fibrotic changes (Supplementary Fig. 2g). 
a

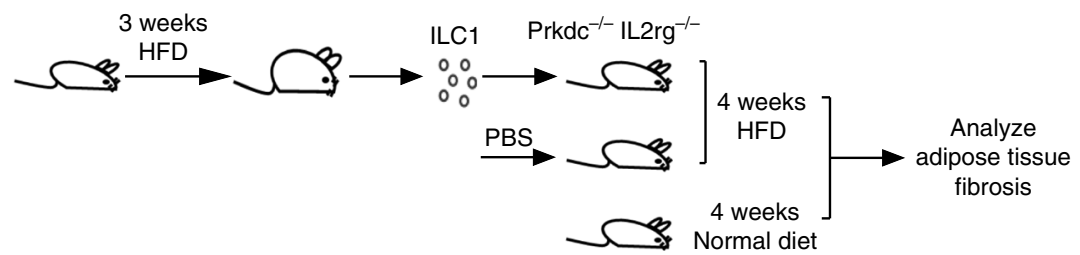

b

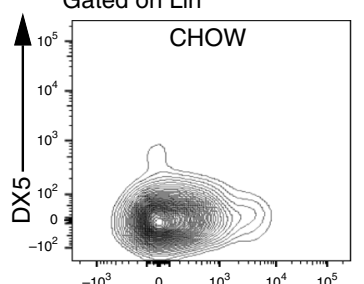

Nkp46

d
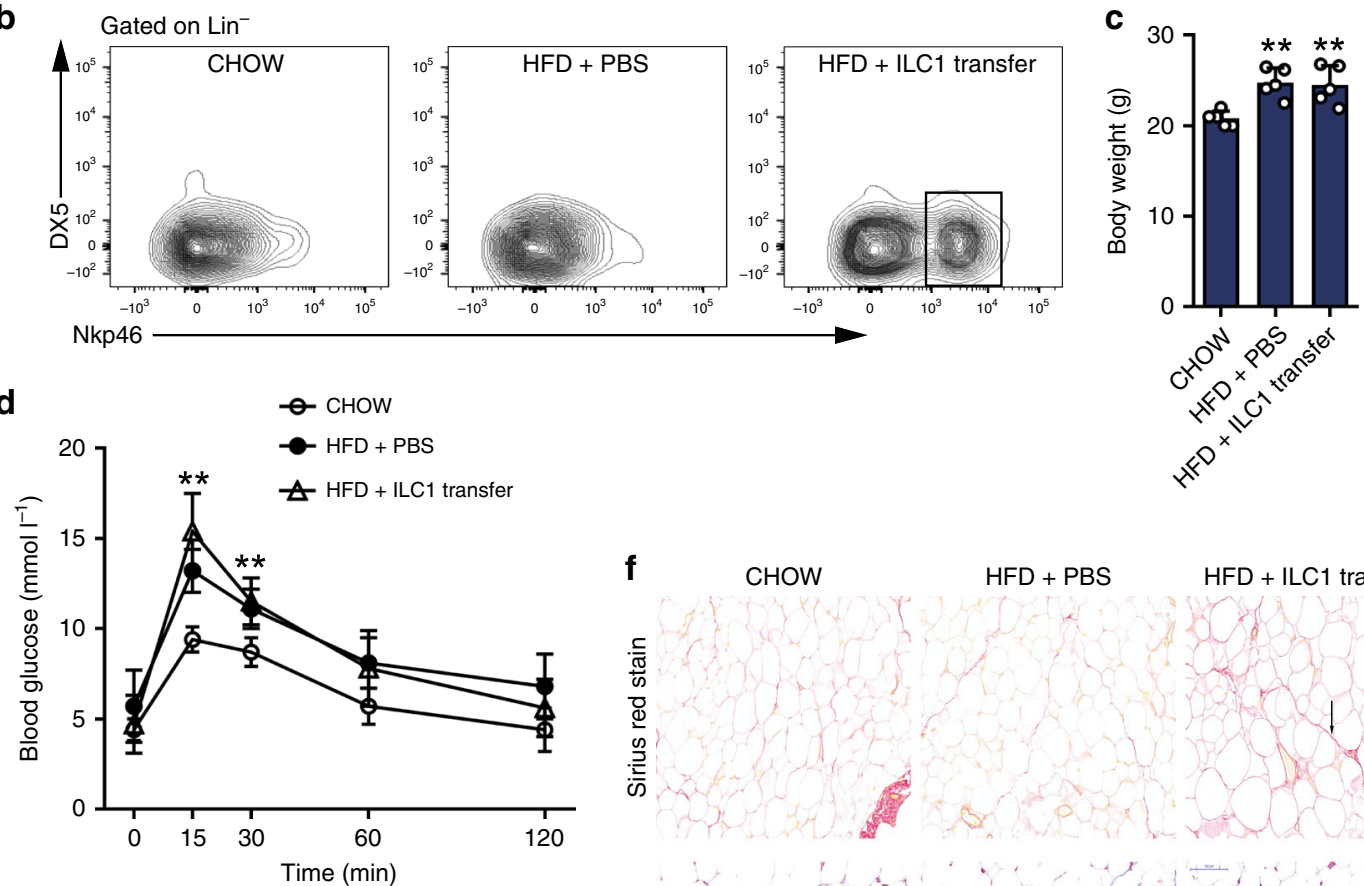

f $\quad$ HFD + PBS HFD + ILC1 transfer

e
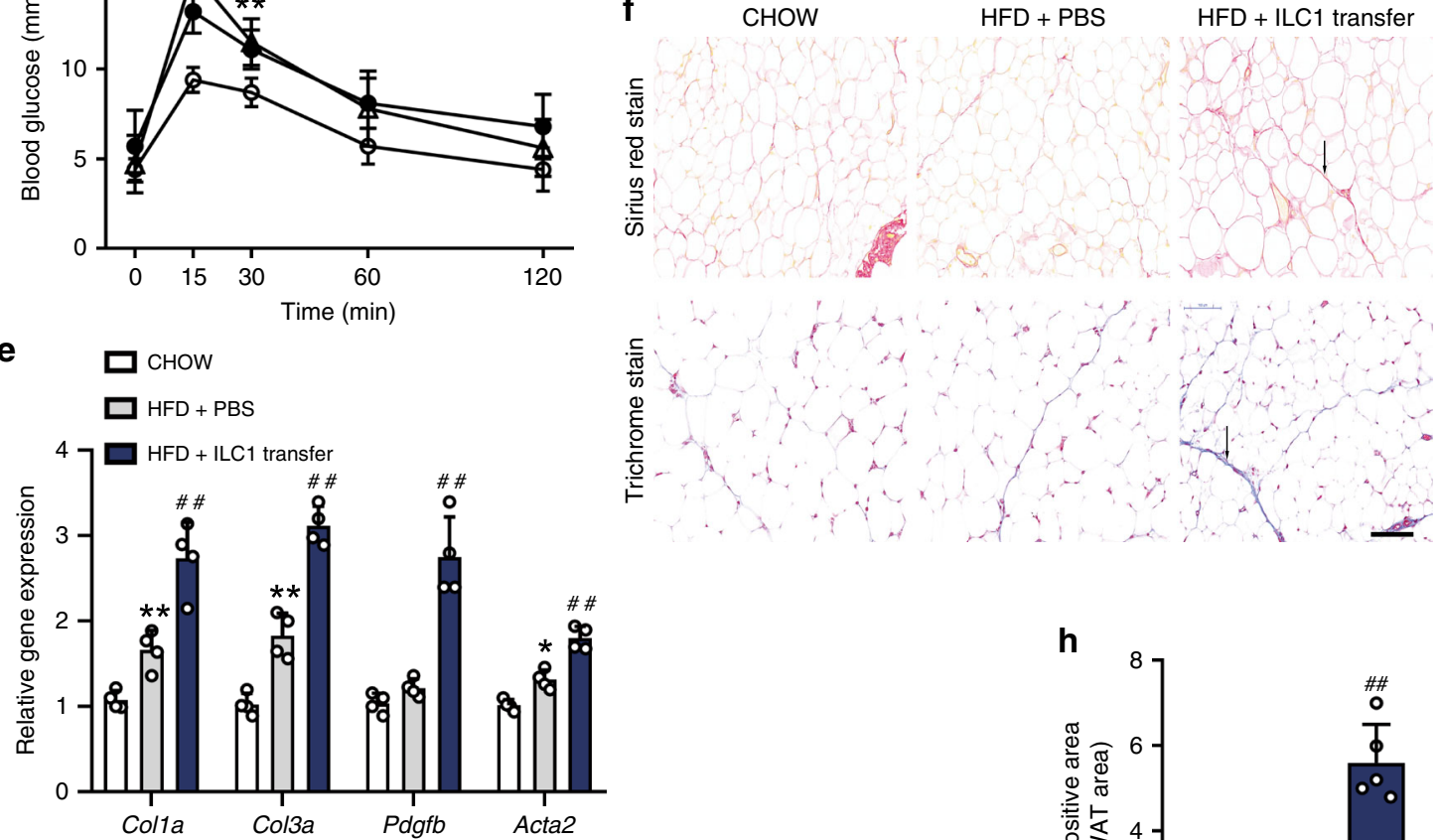

$9 \quad \mathrm{CHOW} \quad \mathrm{HFD}+\mathrm{PBS}$
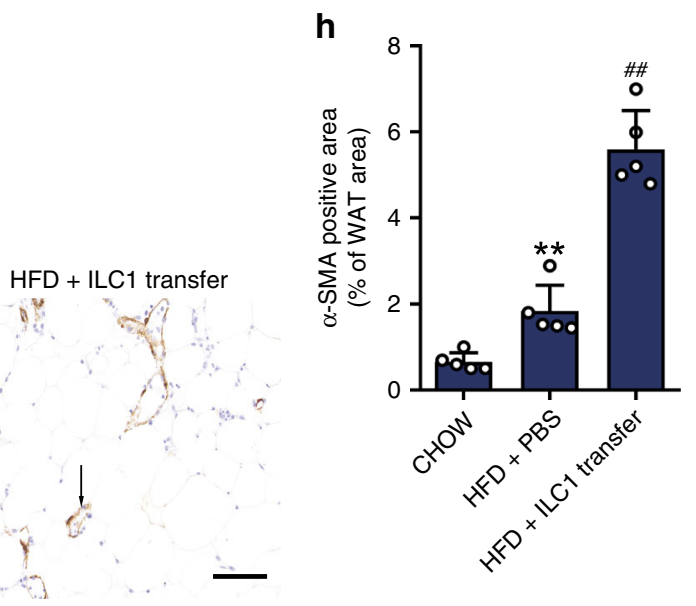

Fig. 3 Adipose ILC1s contribute to obesity-associated adipose fibrosis. Briefly, C57BL/ 6 mice were fed a HFD for 3 weeks, and $5 \times 10^{4}$ adipose ILC1s were

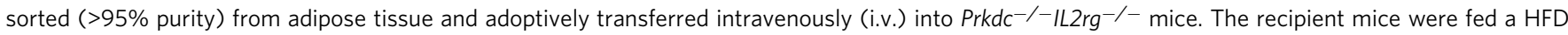
for 4 weeks. a Schematic of the experiment. b Representative plots indicate adoptively transferred adipose ILC1s in the VAT of recipient mice 4 weeks after transfer. c Body weight. d Glucose tolerance test. e Expression levels of fibrosis-related genes, assessed by q-PCR. $\mathbf{f}$ Representative Masson's trichrome $\mathrm{C}$ and Sirius red staining in VAT of mice with different treatment (scale bar: $100 \mu \mathrm{m}$ ). $\mathbf{g}, \mathbf{h}$ Representative $\alpha \mathrm{SMA}$ staining (scale bar: $100 \mu \mathrm{m})$ and quantification of $\alpha S M A$-positive area in VAT of mice with different treatment. Arrows indicate positive staining areas. ${ }^{\star} P<0.05$ vs. $\mathrm{CHOW}$ group; ${ }^{\star \star} P<$ 0.01 vs. CHOW group; $\# P<0.01$ vs. HFD+PBS group (two-way ANOVA tests followed by Bonferroni post hoc test). The data are representative of three independent experiments, with $n=4-6$ mice per group. Error bars indicate s.d. 


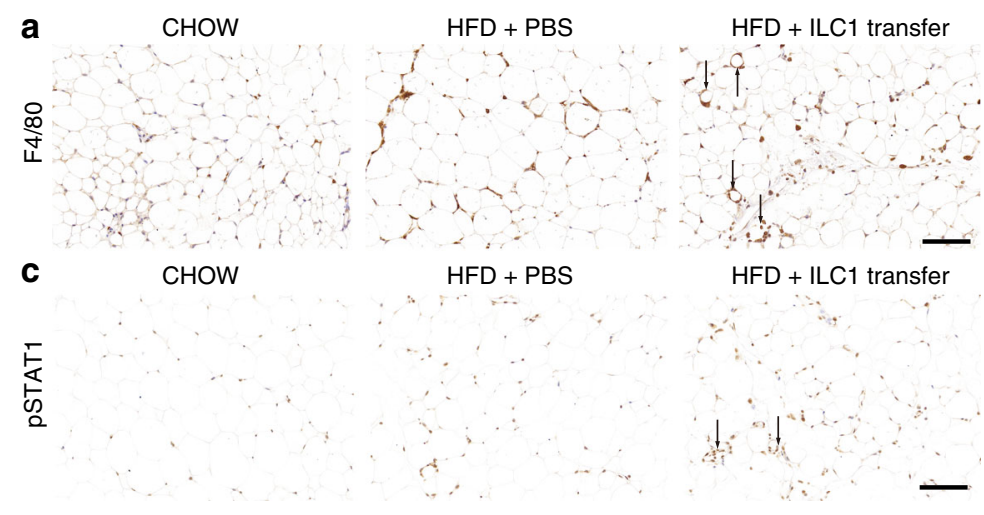

b

d

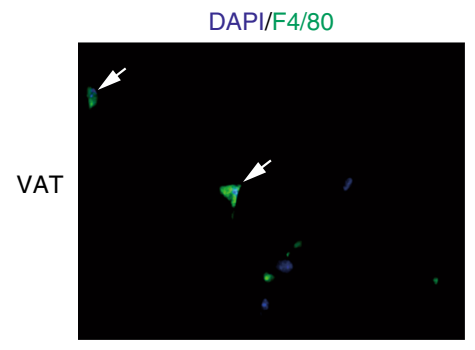

e

g
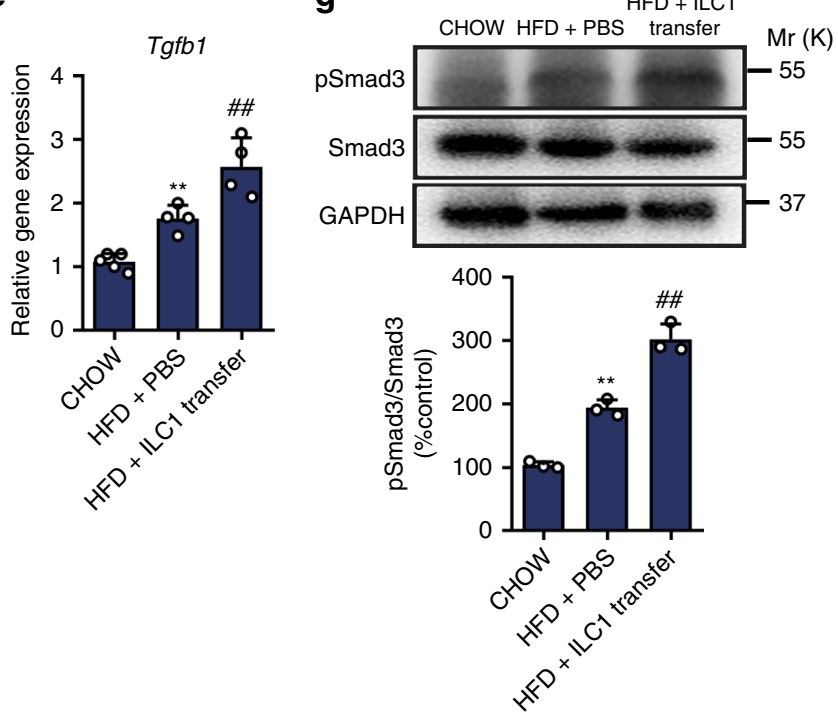

f
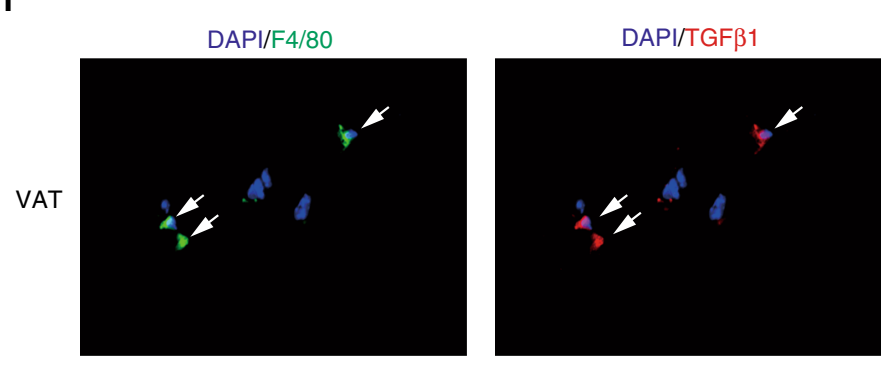

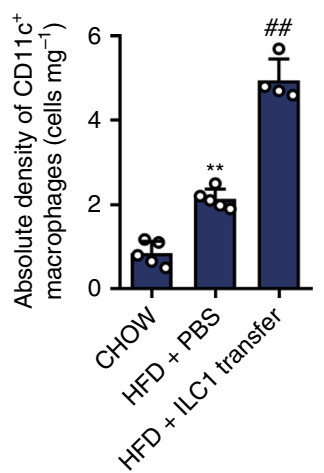

Merge

(DAPI/F4/80/pSTAT1)

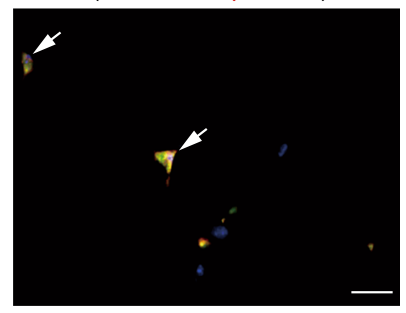

h
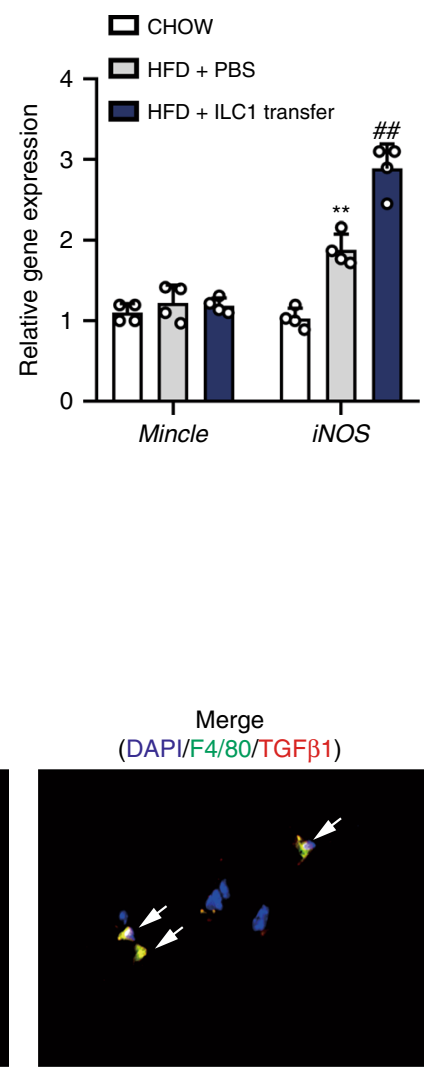

\section{Discussion}

Whether adipose ILCs contribute to the development of obese T2D in humans and the underlying mechanisms remain unclear. In this study, we characterize adipose ILC1s as a pathophysiology factor linking obesity and T2D. More importantly, through a comprehensive set of experiments in humans and mice, we demonstrated that adipose ILC1s promote adipose tissue fibrogenesis via increasing $\mathrm{CD}_{11 \mathrm{c}^{+}}$macrophages and activating the TGF- $\beta 1 /$ Smad3 signaling pathway.

This study provides evidences that adipose ILC1s correlated with the development of obese T2D in humans. In this study, the absolute density (cells $\mathrm{mg}^{-1}$ ) of adipose ILC1s was investigated, since it has been reported that other adipose-resident cells, such as natural killer T (NKT) cells, ILC2, and regulatory T cells (Tregs) are 
Fig. 4 Adipose ILC1s regulate ATMs and activate TGF- $\beta 1$ signaling pathway. C57BL/ 6 mice were fed a HFD for 3 weeks, and $5 \times 10^{4}$ adipose ILC1s were sorted and adoptively transferred into $\mathrm{Prkdc}^{-/-} / \mathrm{L} 2 \mathrm{rg}^{-/-}$mice. The recipient mice were fed a HFD for 4 weeks. a Representative microscopic images with F4/80 staining in VAT of mice with different treatment (scale bar: $100 \mu \mathrm{m}$ ). b Absolute density (cells $\mathrm{mg}^{-1}$ ) of $\mathrm{CD}_{11 \mathrm{c}^{+}}$macrophages was analyzed in the VAT of recipient mice compared with CHOW or HFD controls. c Representative microscopic images with phosphorylated STAT1 staining in VAT of mice with different treatment (scale bar: $100 \mu \mathrm{m}$ ). d VAT specimens of mice receiving ILC1 transfer were costained for F4/80 (green), pSTAT1 (red), and DAPI (blue). Colocalization of F4/80 and pSTAT1 is shown in yellow in the merged image (scale bar: $20 \mu \mathrm{m}$ ). White arrows indicate typical stained cells. e Relative mRNA expression of Tgfb1. f VAT specimens of mice receiving ILC1 transfer were costained for F4/80 (green), TGF- $\beta 1$ (red), and DAPI (blue). Colocalization of F4/80 and TGF- $\beta 1$ is shown in yellow in the merged image (scale bar: $20 \mu \mathrm{m}$ ). White arrows indicate typical stained cells. $\mathbf{g}$ Phosphorylation and total levels of Smad3 in VAT of mice with different treatment. The phosphorylation levels of Smad3 were normalized to endogenous Smad3 levels, and are expressed as a percentage to those of CHOW group. h Relative mRNA expression of Mincle and iNOS. ${ }^{\star \star} P<0.01$ vs. CHOW group; $\# \# P<0.01$ vs. HFD+PBS group (two-way ANOVA tests followed by Bonferroni post hoc test). The data are representative of three independent experiments, with $n=4-6$ mice per group. Error bars indicate s.d.

reduced in the VAT of HFD-fed mice and affect the frequencies of minor cell populations ${ }^{20}$. A role of adipose ILC1s in the development of T2D was elucidated by the findings that adipose tissue of obese T2D patients contained significantly higher numbers of adipose ILC1s than that of obese and control subjects (Fig. 1), and further reinforced by correlations of adipose ILC1s with glycemic parameters either before or after adjustments for confounding factors (Table 2). Notably, a tight association was observed between adipose ILC1s and circulating ILC1s, with the reduction of circulating ILC1s positively correlated with the improvement of glycemic parameters in obese T2D patients after 3 months of bariatric surgery (Fig. 1). These data also indicate that increased levels of circulating ILC1s might act as a circulating marker of adipose dysfunction and T2D. These data expand our knowledge of adipose ILC1s and provide clinical evidences that adipose ILC1s contribute to the development of obese T2D in humans.

Another important finding of this study is the characterization of a previously unrecognized role of adipose ILC1s in adipose tissue fibrogenesis. Our data showed that adipose ILC1s positively correlated with extents of fibrosis in human omental adipose tissue and represented a major contributor to adipose tissue fibrogenesis as depicted in a multivariate regression model (Fig. 2, Table 3). Importantly, a direct role of adipose ILC1s in adipose tissue fibrogenesis was observed in our in vitro co-culture experiments, in which SVFs from control subjects were cocultured with either $\mathrm{CD} 45^{+}$cells or ILCs depleted $\mathrm{CD} 45^{+}$cells sorted from the SVFs of obese T2D patients (Fig. 2). To exclude the role of CD8 ${ }^{+} \mathrm{T}$ cells and NK cells which have been reported to express IFN- $\gamma$ in obese VAT ${ }^{21,22}$, adipose ILCs were magnetically enriched from obese T2D patients and co-cultured with SVFs of control subjects. Expressions of fibrosis-related genes and ECM regulators were significantly increased in SVFs after cocultured with ILCs, indicating that adipose ILC1s can directly promote adipose tissue fibrogenesis and are independent of other obesity-induced $\mathrm{CD} 45^{+}$cells (Fig. 2). Of note, administration of neutralizing IFN- $\gamma$ antibody markedly reversed the upregulation of genes related to fibrogenesis in SVFs co-cultured with adipose ILCs (Fig. 2), further confirming that IFN- $\gamma$ production mediates the effect of adipose ILC1s on adipose tissue fibrogenesis. In addition, since IFN- $\gamma$ expression from adipose ILC1s was similar between obese subjects and obese T2D patients (Fig. 2), coculture experiments using ILCs from SVFs of obese subjects were expected to obtain similar results to obese T2D patients. Therefore, no further co-culture experiments were performed. In support of our findings in humans, adoptive transfer of adipose ILC1s into Prkdc $\mathrm{C}^{-/} \mathrm{Il} 2 \mathrm{rg}^{-/-}$mice aggravated adipose tissue fibrosis in the absence of complicated influences of $\mathrm{T}$ cells and NK cells, further demonstrating that adipose ILC1s play a causative role in the development of adipose tissue fibrosis (Fig. 3).

To validate that adipose ILC1s-derived IFN- $\gamma$ was responsible for adipose tissue fibrogenesis and glycemic intolerance during
HFD feeding, adipose ILC1s from HFD-fed Ifng ${ }^{-/-}$mice or wildtype mice were adoptively transferred into recipient mice. Consistently, mice receiving ILC1 transfer showed higher fasting glucose level, more severe glycemic intolerance, and aggravated adipose fibrosis compared with HFD mice receiving PBS injection (Fig. 5). However, following Ifng-/- ILC1 transfer into recipients, no significant differences were found in these glycemic and adipose fibrosis parameters compared with HFD mice receiving PBS injection (Fig. 5). In addition, we utilized antibody depletion methods to inhibit adipose ILC1s accumulation and observed a notably remission of adipose fibrosis, suggesting the potential of targeting adipose ILC1s to improve adipose tissue fibrosis and glycemic intolerance in obesity (Fig. 6). Importantly, these beneficial effects of IL-12 neutralizing antibody treatment were also observed in intact wild-type mice, not only in immunodeficient mice (Supplementary Fig. 2). Collectively, these results provided direct and solid evidences supporting the role of IFN- $\gamma$ production by adipose ILC1s in promoting adipose tissue fibrogenesis and provide insights into the pathogenic mechanism of local immune disturbances in obesity-associated metabolic disturbance.

Recently, ATMs are reported to play a key role in regulating adipose tissue fibrogenesis ${ }^{16-18}$, promoting us to explore the role of adipose ILC1s in regulating ATMs function in humans. We found that $\mathrm{CD}_{11 \mathrm{c}^{+}}$macrophages were increased in SVFs co-cultured with $\mathrm{CD} 45^{+}$cells containing adipose ILC1s (Fig. 2), thus providing evidences that adipose ILC1s could regulate CD11c ${ }^{+}$macrophage activation in humans. A recent study using IFN- $\gamma$ reporter mice showed that IFN- $\gamma$ is predominantly produced by ILC1s in adipose tissue, via which adipose ILC1s promote proinflammatory CD11 $\mathrm{c}^{+}$ macrophage activation ${ }^{11}$. Consistent with these previous findings ${ }^{17}$, increased $\mathrm{CD} 11 \mathrm{c}^{+}$and $\mathrm{CD} 11 \mathrm{c}^{+} \mathrm{CD} 206^{+}$macrophages, and CLS density were observed in VAT of mice receiving ILC1 transfer (Fig. 4). More importantly, we provided evidences that adipose ILC1 from obese subjects expressed high levels of IFN- $\gamma$ (Fig. 2). The role of adipose ILC1-derived IFN- $\gamma$ in macrophage activation was further reinforced by the in vitro co-culture experiments, in which adipose ILC1s were found to promote BMDMs activation toward proinflammatory phenotype, depending on IFN- $\gamma$ production (Supplementary Fig. 2). TGF- $\beta 1 /$ Smad3 activation contributes to persistent and aberrant ECM remodeling of VAT in obesity, and modulation of TGF- $\beta 1$ activity might be an effective treatment strategy for obesity and related diabetes ${ }^{23,24}$. In line with previous studies that macrophage infiltrated areas are in rich of TGF- $\beta 1$ expression in VAT of obese mice ${ }^{23}$, VAT of mice receiving ILC1 transfer showed increased mRNA expression of $T g f b 1$ (Fig. 4). Accordingly, activated TGF- $\beta 1$ pathway, as evidenced by increased phosphorylation of pSmad3, was also observed (Fig. 4). Importantly, our in vitro co-culture experiments also delineate that adipose ILC1 act to increase Tgfb1 expression in SVFs (Fig. 2). These data thus demonstrate that induction of adipose tissue 
a

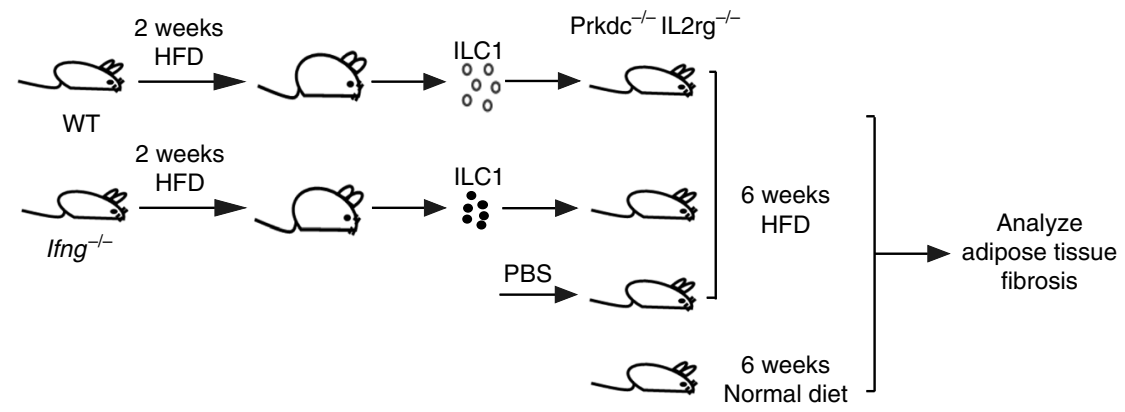

b
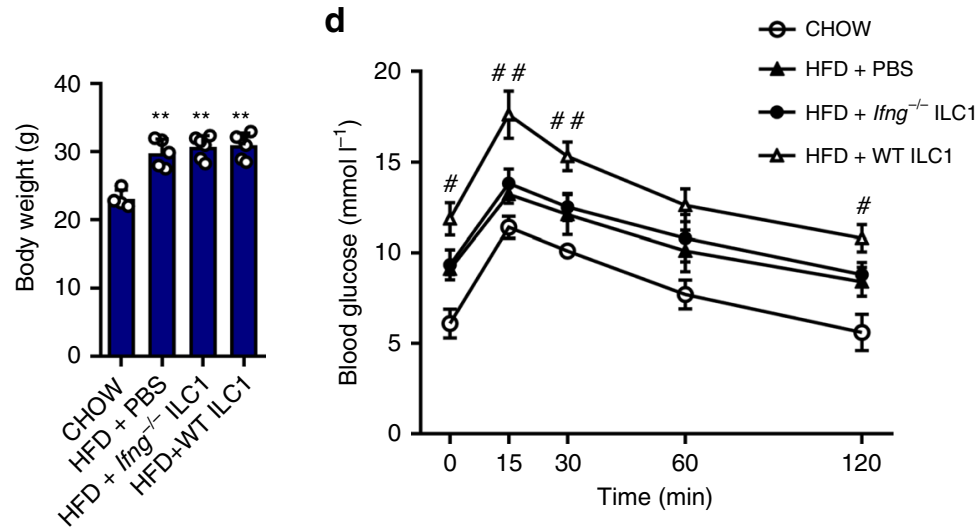

C

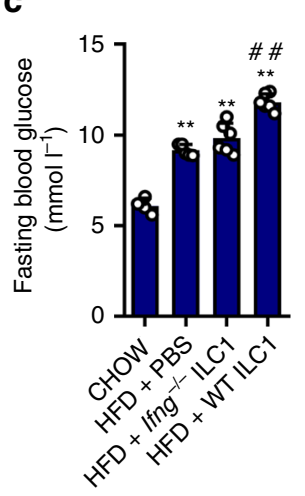

e $\square$ CHOW $\square \mathrm{HFD}+$ lfng $^{-/}$ILC1
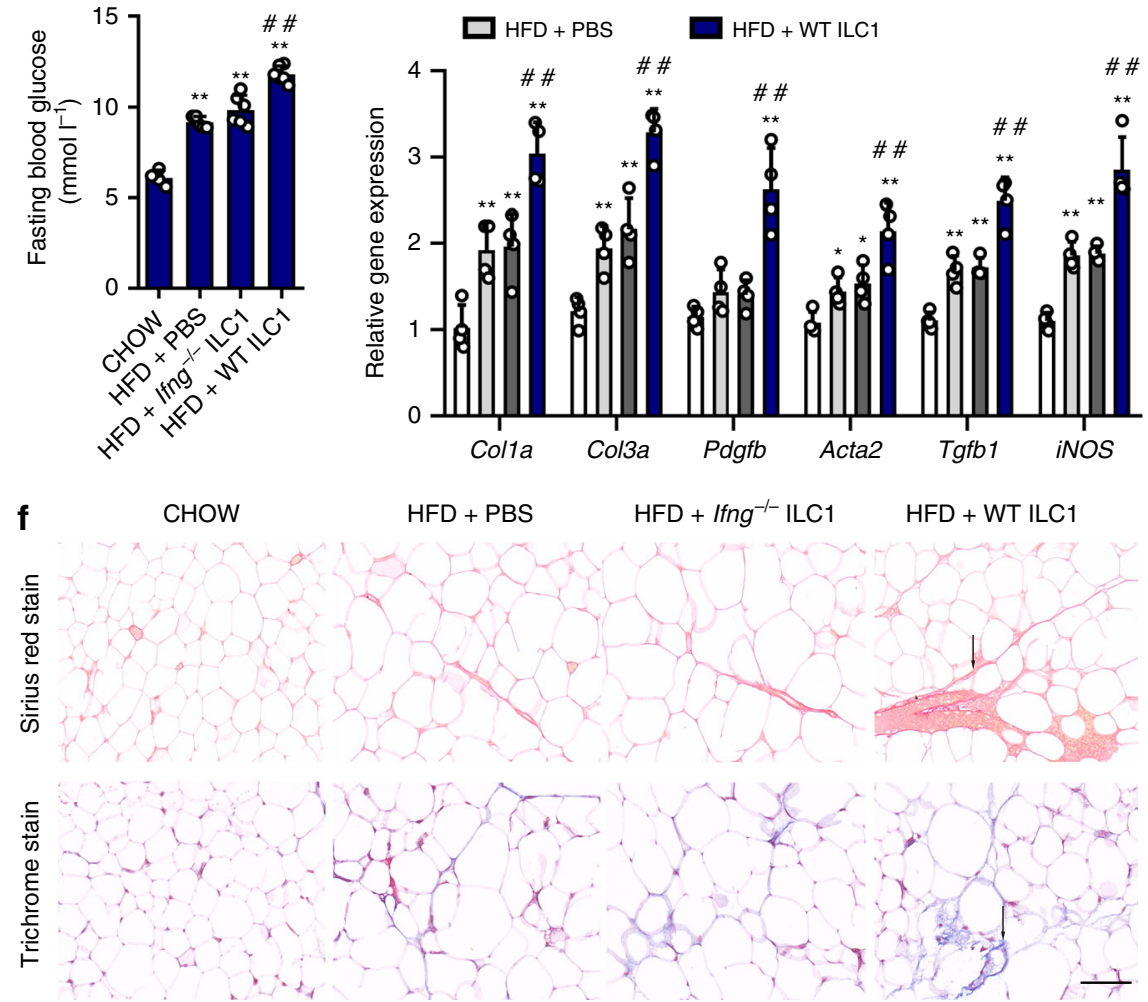

$\mathrm{HFD}+\mathrm{PBS} \quad \mathrm{HFD}+I_{\mathrm{Ing}}{ }^{-/-} \mathrm{ILC} 1$

HFD + WT ILC1

Fig. 5 Adipose ILC1s induce adipose fibrosis through IFN- $\gamma$ production. C57BL/6 and Ifng-/- mice were fed a HFD for 2 weeks, and $5 \times 10^{4}$ adipose ILC1s

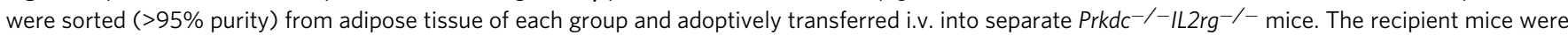
fed a HFD for 6 weeks. Three weeks after HFD, adipose ILC1s were adoptively transferred into recipients again. a Schematic of the experiment. b Body weight. c Fasting blood glucose. d Glucose tolerance test. e Expression levels of fibrosis-related genes, assessed by q-PCR. $\mathbf{f}$ Representative Masson's trichrome $C$ and Sirius red staining in VAT of mice with different treatment (scale bar: $100 \mu \mathrm{m}$ ). Arrows indicate positive staining areas. ${ }^{*} P<0.05$ vs. CHOW group; ${ }^{\star \star} P<0.01$ vs. CHOW group; $\# P<0.05$ vs. HFD+Ifng ${ }^{-/-}$ILC1 group; \#\# $P<0.01$ vs. HFD+lfng ${ }^{-/}$ILC1 group (two-way ANOVA tests followed by Bonferroni post hoc test). The data are representative of two independent experiments, with $n=4-6$ mice per group. Error bars indicate s.d. 
a

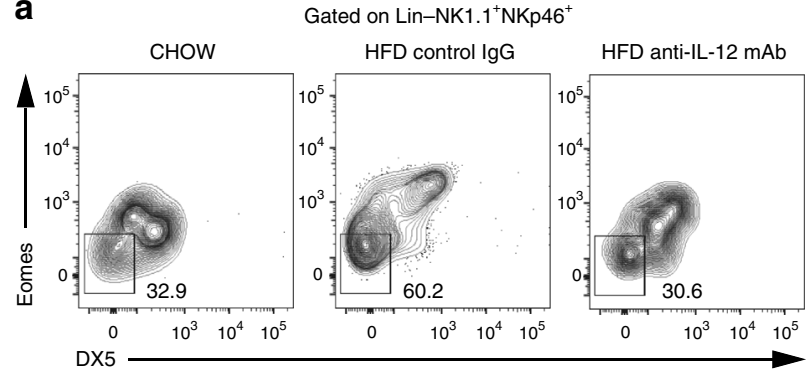

d
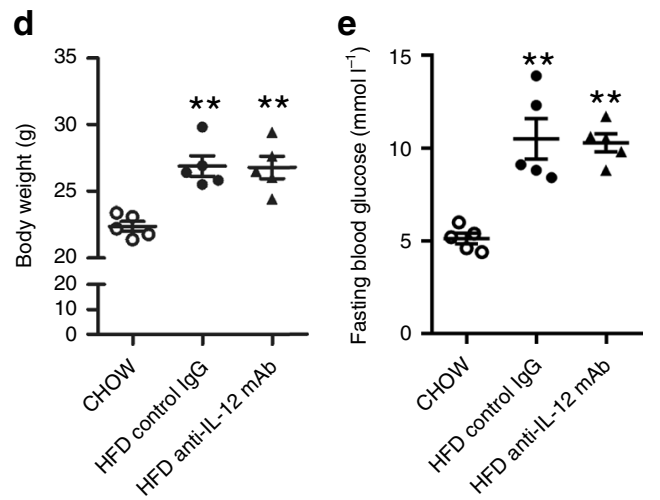

g
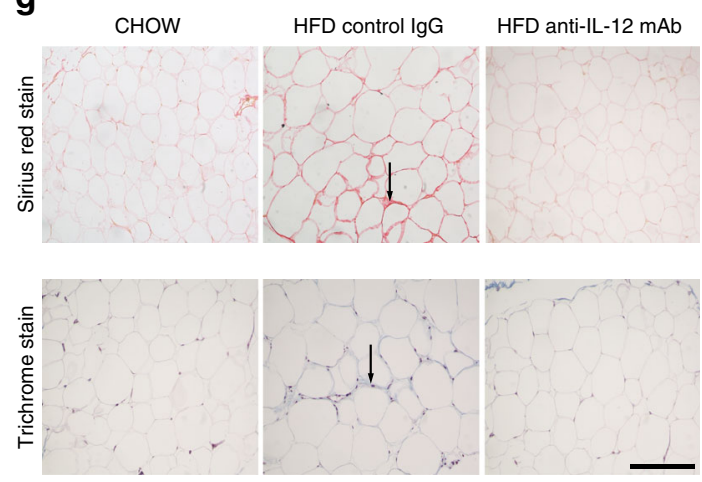

i

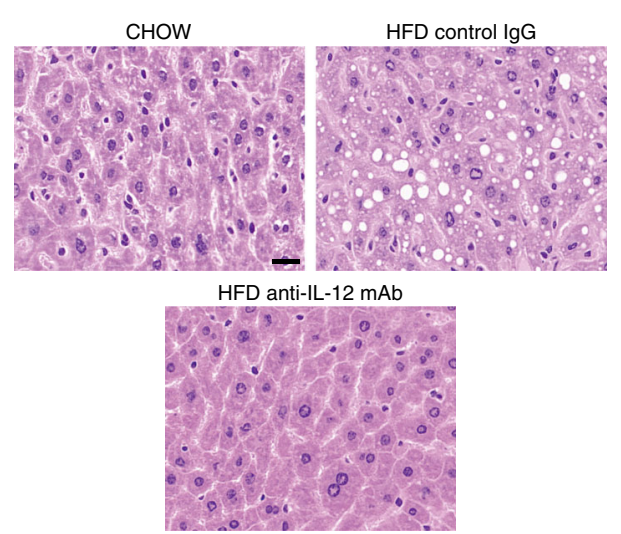

fibrosis by adipose ILC1 is related to the regulation of macrophage and activation of the TGF- $\beta 1 / \mathrm{pSmad} 3$ signaling pathway.

Fibrogenesis is a complex process caused by a variety of cells, such as myofibroblasts, macrophages, and parenchymal cells. Previous studies showed that myofibroblasts are derived from different cell types including resident fibroblasts and fibrocytes, depending on different tissues or organs ${ }^{25}$. In adipose tissue, interaction
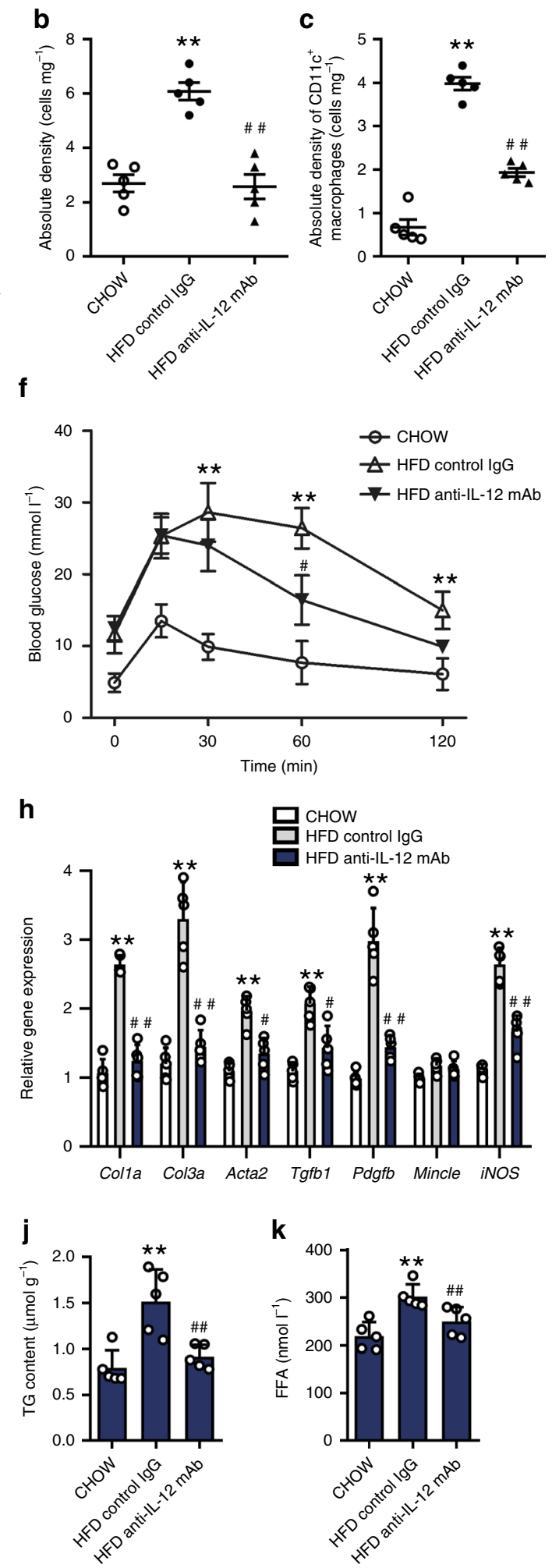

between Mincle expressing macrophages and fibroblast might promote myofibroblast formation ${ }^{17}$. In our in vitro co-culture experiments, expression of Mincle was increased after stimulation with trehalose-6,6'-dimycolate and palmitate in SVFs co-cultured with $\mathrm{CD}_{4}{ }^{+}$immune cells containing adipose ILC1s and in SVFs co-cultured with adipose ILCs (Fig. 2). Furthermore, increased number of aSMA-positive cells or myofibroblasts was observed in 
Fig. 6 Inhibiting adipose ILC1s accumulation attenuates adipose fibrosis. Rag1-/- mice were fed with normal diet or HFD, while mice in the HFD group were randomized into two groups of HFD anti-IL-12 mAb group and HFD control IgG group and continued with HFD feeding for another 4 weeks. HFD anti-IL-12 $\mathrm{mAb}$ group received rat anti-murine anti-IL-12p35 mAb administration via intraperitoneal injection at $250 \mu \mathrm{g}$ per mouse every 3 days for 4 weeks; HFD control IgG group received the same dose of control IgG. a Representative plots indicate adipose ILC1s in the VAT of mice with different treatment. b Absolute density (cells $\mathrm{mg}^{-1}$ ) of adipose ILC1s in VAT. c Absolute density of CD11c macrophages (cells mg ${ }^{-1}$ ) in VAT. d Body weight. e Fasting blood glucose. $\mathbf{f}$ Glucose tolerance test. $\mathbf{g}$ Representative Masson's trichrome $\mathrm{C}$ and Sirius red staining in VAT of mice with different treatment (scale bar: 100 $\mu \mathrm{m})$. Arrows indicate positive staining areas. $\mathbf{h}$ Expression levels of fibrosis-related genes, assessed by q-PCR. $\mathbf{i}$ Representative microscopic images with H\&E staining in the liver (scale bar: $20 \mu \mathrm{m}$ ). j, k Hepatic triglyceride levels and serum free fatty acid (FFA) levels. ${ }^{* \star} P<0.01$ vs. CHOW group; ${ }^{\#} P<0.05$ vs. HFD+control lgG group; $\# \#<<0.01$ vs. HFD+control lgG group (two-way ANOVA tests followed by Bonferroni post hoc test). The data are representative of two independent experiments, with $n=5$ mice per group. Error bars indicate s.d.

mice receiving ILC1s transfer (Fig. 3). Besides myofibroblasts, preadipocytes are also reported to be source of the ECM, and macrophage can increase the profibrogenesis function through $i N O S$ expression and subsequent production of $\mathrm{NO}^{16,18}$. Here, increased expression of $i N O S$ in both SVFs co-cultured with adipose ILC1s and VAT of mice receiving ILC1s transfer were observed (Figs. 2, 4). Hence, these findings indicate that both preadipocytes and myofibroblasts participate in the process of adipose ILC1sassociated fibrogenesis. In addition to macrophages, iNOS can be expressed by a variety of immune cell populations after induction by cytokines or other stimuli, including neutrophils ${ }^{26}$, mature dendritic cells ${ }^{27}$, and Th17 cells ${ }^{28}$, which have been reported to be markedly increased in obese subjects ${ }^{5}$. In this study, since the gene expression of $i N O S$ was detected using adipose tissue homogenates rather than sorted $\mathrm{CD} 11 \mathrm{c}^{+}$macrophages, the iNOS reduction not entirely in accordance with the decrease of CD11c ${ }^{+}$macrophage might due to other cell types' contributions to iNOS expression in anti-IL-12 mAb-treated mice.

A recent study showed that the frequency of a subset of CD56 ${ }^{\text {low }}{ }^{-} D 3^{-}{ }^{-} D 16^{-}$CD $127^{\text {low }}$ ILC1-like cells was decreased in adipose tissue of extremely obese subjects ${ }^{29}$. Based on the limited clinical baseline data provided by that study, different extent of obesity (mean BMI: 48 vs. 37 ) and glycemic status (non T2D vs. T2D) of obese subjects might account for the discrepancy on human adipose ILC1s between that study and our study, apart from the different markers in identification of adipose ILC1s. To reach a consensus on ILC1 identification and better clarify its role in human metabolic disease, follow-up studies including a larger clinical cohort with different stage of obesity and a strictly matched control cohort are required to evaluate the phenotypic and functional characteristics of adipose ILC1s.

In conclusion, our data clarify a direct role of adipose ILC1s in adipose tissue fibrogenesis and provide clues for understanding the pathogenic mechanism of local immune disturbances in obesity-associated metabolic disturbance. More importantly, our findings reveal that adipose ILC1s contribute to the development of obese T2D, thus providing a pathophysiological link between obesity and T2D. Adipose ILC1s may thus serve as a therapeutic target for the treatment or prevention of obese T2D.

\section{Methods}

Patients. A total of 85 individuals, including 49 obese patients underwent laparoscopic Roux-en-Y gastric bypass (RYGB) surgery, and 36 age- and sex-matched nonobese non-T2D controls received elective abdominal surgery (e.g., hernia or hemangioma resection) were enrolled from April 2017 to December 2017 at Drum Tower Hospital Affiliated to Nanjing University Medical School. All the enrolled 49 obese subjects had no history of diabetes and they received oral glucose tolerance test before surgery, in which 22 individuals were diagnosed as T2D according to American Diabetes Association criteria ${ }^{30}$. All participants completed questionnaires for medical history assessment and underwent anthropometric measurements. Patient height was measured by stadiometer with shoes off, to the nearest centimeter. The weight was measured on a digital scale with identical light clothing on, to the nearest $0.1 \mathrm{~kg}$. The BMI was calculated as weight divided by height squared. Exclusion criteria were: acute infection in the past 3 months; stroke or myocardial infarction; autoimmune diseases, e.g., rheumatoid arthritis and systemic lupus erythematosus; malignant tumors; chronic digestive diseases, e.g., Crohn's disease and
}

colitis gravis; abnormal liver or renal dysfunction, with alanine transaminase (ALT) or aspartate transaminase (AST) 2.5-folds higher than the normal range, or estimated glomerular filtration rate (eGFR) $<60 \mathrm{ml} \mathrm{min}^{-1} 1.73 \mathrm{~m}^{-2}$, based on the CKD-EPI equation; use of nonsteroidal or steroidal anti-inflammatory medicines in past 3 months; pregnancy; other endocrine system diseases, e.g., thyroid diseases and Cushing's syndrome. Obese patients who underwent bariatric surgery were followedup, and blood samples were collected from 19 obese patients and 17 obese T2D patients 3 months after surgery at the end of the study.

This study, including the use of human tissues was approved by the Ethics Review Committee of Nanjing Drum Tower Hospital Affiliated to Nanjing University Medical School (Approval number: 2017-030-02). All patients and control individuals meeting the inclusion criteria gave consent at the time of enrolment.

Animal studies. All animal experimental protocols were approved by the Research Animal Care Committee of Drum Tower Hospital Affiliated to Nanjing University Medical School (Approval number: 2019AE01002), Nanjing, China. The following strains were used in this study: C57BL/6, Rag1 ${ }^{-/-}$, Ifng $^{-/-}$(B6.129S7-Ifngtm1Ts/J, stock number 002287, purchased from the Jackson Laboratory), and Prkdc ${ }^{-1} \mathrm{IL}_{2 \mathrm{rg}^{-}}-$ (NOD background, lacking mature T, B, and natural killer lymphocytes) mice. Experiments were conducted using age- and gender-matched mice in accordance with approved institutional protocols.

For antibody treatment experiments, 6-week-old male Rag1 ${ }^{-/-}$and C57BL/6 mice were obtained from Nanjing Biomedical Research Institute of Nanjing University and housed in a controlled environment (12 -h daylight cycle, lights off at 18:00) with food and water ad libitum. After 1 week of acclimation on a normal diet, mice were randomly divided into two groups and fed either a normal diet or a HFD (D12492, Research Diets). After 1 week, mice in the HFD group were randomized into two groups of HFD anti-IL-12 mAb group and HFD control IgG group, and continued with HFD feeding for another 4 weeks. HFD anti-IL-12 mAb group received rat anti-murine anti-IL-12p35 mAb (eBioscience, C18.2) administration via intraperitoneal injection at $250 \mu \mathrm{g}$ per mouse every 3 days for 4 weeks; HFD control IgG group received the same dose of control IgG.

In adoptive transfer experiments, adipose ILC1s were purified from adipose tissue from C57BL/6 or Ifng ${ }^{-/-}$mice fed a HFD by a flow cytometer (FACSAriaIII,

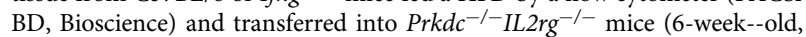
purchased from Biocytogen Co., Ltd, Beijing, China) on HFD. After HFD feeding, recipient peripheral organs were harvested, and adoptively transferred cells were analyzed by flow cytometry.

All mice were weighed at the beginning to the feeding period and weekly thereafter until killed. Glucose tolerance test (GTT) was performed with an intraperitoneal injection of $10 \% \mathrm{D}$-glucose solution $\left(2 \mathrm{~g} \mathrm{Kg}^{-1}\right.$ body weight) into the mice. Blood glucose levels were measured at $0,15,30,60$, and $120 \mathrm{~min}$ after glucose administration. Two days after GTT, all animals were anesthetized in chambers saturated with isoflurane and killed. Blood samples, VAT, and liver tissues were collected.

Blood sample analysis. Fasting blood samples were also collected for HbAlc, triglycerides, total cholesterol, high-density lipoprotein cholesterol (HDL-C), lowdensity lipoprotein cholesterol (LDL-C), and free fatty acid (FFA) measurements. Plasma glucose amounts were assessed by the hexokinase method on TBA-200FR (Tokyo, Japan). Insulin concentration was evaluated by a chemiluminescence immunoassay (Cobas e601, Roche). HbAlc was quantitated by high-performance liquid chromatography (Bio-Rad D-10). Triglyceride, total cholesterol, HDL-C, and LDL-C concentrations were assessed by specific immunoassays (Cobas e601, Roche). Serum FFAs were detected via an enzymatic method (DiaSys Diagnostic Systems, Co., Ltd). HOMA-IR was derived as follows: [fasting serum insulin $(\mathrm{mU} / \mathrm{l}) \times$ fasting glucose $(\mathrm{mmol} / \mathrm{l})] / 22.5^{31}$. The Adipo-IR was defined as fasting insulin concentration $(\mathrm{mIU} / \mathrm{ml}) \times$ fasting FFA $(\mathrm{mmol} / \mathrm{l})^{32,33}$.

Mouse serum was analyzed for FFA using a standard enzymatic assay following the manufacturer's protocols (ColorfulGene biological technology Co., Ltd, Wuhan, China).

Measurement of triglyceride content in the liver. For mouse liver triglyceride analysis, triglyceride content in the harvested liver tissue was measured using an enzymatic assay kit following the manufacturer's protocols (JYM0190Mo, ColorfulGene biological technology Co., Ltd, Wuhan, China). 
Peripheral blood mononuclear cell isolation. PBMCs were isolated with vacutainer cell preparation tubes (Becton Dickinson, Franklin Lakes, NJ) from fasting blood samples in the non-obese and obese patients pre-surgery and in the obese patients after 3 months follow-up of surgery. Briefly, after centrifugation at $1800 \times$ $\mathrm{g}$ for $30 \mathrm{~min}$, cells in the resulting supernatants were collected, washed with phosphate-buffered saline (PBS), and frozen gradually within $8 \mathrm{~h}$ in freezing buffer containing $10 \%$ DMSO and $90 \%$ fetal bovine serum (FBS). Samples were stored in liquid nitrogen until further analysis.

Adipose tissue biopsy and SVF preparation. Periumbilical adipose tissue samples at the omental region were obtained from 21 obese individials, 20 obese T2D patients, and 24 non-obese non-T2D control individuals perioperatively. Adipose tissue samples $(\sim 10 \mathrm{~g})$ were collected and transported to laboratory immediately. For human SVFs preparation, fresh adipose samples were cut into small pieces and digested with $0.1 \%$ type II collagenase (Sigma-Aldrich, USA). After incubation for $1 \mathrm{~h}$ with shaking on a MACSmix Tube Rotator, the specimens were filtered through a $100-\mu \mathrm{m}$ nylon mesh and centrifuged at $500 \times g$ for $10 \mathrm{~min}$. The resulting supernatants were aspirated, and the pellets were resuspended in red blood cell (RBC) lysis buffer (GE, USA)

Mouse SVFs were prepared using $0.1 \%$ type I collagenase (Sigma-Aldrich, USA) ${ }^{23}$. In brief, VAT was physically dissociated using scissors and incubated for $1 \mathrm{~h}$ in digest solution $(1 \mathrm{mg} / \mathrm{ml}$ type I collagenase in RPMI supplemented with $5 \%$ fetal calf serum, $1 \%$ L-glutamine, $1 \%$ penicillin-streptomycin, and $10 \mathrm{mM}$ HEPES). Resulting dissociated tissue was passed through $100-\mu \mathrm{m}$ nylon mesh, centrifuged, and adipocytes were removed from the supernatant. Red blood cells were lysed using RBC lysis buffer.

Flow cytometry. Human PBMCs and SVFs were stained with the following antibodies for flow-cytometry analysis: CD3 (UCHT1, BD Biosciences, 1:200), CD19 (HIB19, BD Biosciences, 1:200), CD16 (B73.1, BD Biosciences, 1:100), CD45 (2D1, BD Biosciences, 1:100), CD127 (HIL-7R-M21, BD Biosciences, 1:50), CD117 (YB5, B8, BD Biosciences, 1:50), CRTH2 (BM16, BD Biosciences, 1:50), NKP44 (p44-8, BD Biosciences, 1:50), CD206 (19.2, BD Biosciences, 1:50), CD14 (M5E2, BD Biosciences, 1:100), IFN- $\gamma$ (4S.B3, BD Biosciences, 1:50), CD11c (Bu15, BioLegend, 1:50), CD5 (L17F12, BioLegend, 1:100), TCR $\alpha \beta$ (IP26, BioLegend, 1:100), and FCERIA (AER-37, BioLegend, 1:100). Human ILC1s were identified as a lineage negative for CD3, CD19, CD16, CD11c, CD5, TCR $\alpha \beta$ and FCERIA $\left(\mathrm{Lin}^{-}\right), \mathrm{CD} 45^{+}, \mathrm{CD} 127^{+}, \mathrm{CD} 117^{-}$, NKP44 ${ }^{-}$, CRTH2 ${ }^{-}$. Human macrophages were identified with antibodies against CD45, CD14, CD11c, and CD206 ${ }^{34}$. 7-aminoactinomycin D (BD Biosciences) was used to gate live cells. For IFN- $\gamma$ detection, intracellular cytokine staining was performed with the Cytofix/Cytoperm Plus kit (BD Biosciences). For animal studies, cell surface staining was performed using the following fluorophore-conjugated antibodies: TCR $\beta$ (H57-597, BD Biosciences, 1:100), CD3 (17A2, BD Biosciences, 1:200), CD19 (1D3, BD Biosciences, 1:200), TCR $\gamma \delta$ (GL3, BD Biosciences, 1:100), Ly-6G (1A8, BD Biosciences, 1:100), F4/80 (BM8, BioLegend, 1:100), NK1.1 (PK136, BD Biosciences, 1:50), NKP46 (29A1.4, BD Biosciences, 1:50), DX5 (DX5, BD Biosciences, 1:50), Eomes (1219A, R\&D Systems, 1:50), T-bet (4B10, BD Biosciences, 1:50), CD11b (M1/70, BD Biosciences, 1:100), CD11c (N418, BD Biosciences, 1:100), CD206 (C068C2, BioLegend, 1:100), CD45 (A20, BD Biosciences, 1:200). For analysis of cell surface molecules, cells were washed with PBS and resuspended in FACS buffer (2\% FBS, 2 mM EDTA, $0.05 \% \mathrm{NaN}_{3}$ in PBS), following by staining with the antibodies indicated in figures in FACS buffer for 30 min at $4{ }^{\circ} \mathrm{C}$. After washing, the stained cells were analyzed on an LSR II Fortessa flow cytometer (BD, Bioscience) or sorted on a FACSAriaIII flow cytometer (BD Biosciences); the data were analyzed with FACSDiva software (BD, Bioscience) or Flowjo software version 9.6.4 (Tree Star, Inc.). The gating strategy for flowcytometry analysis is shown in Supplementary Fig. 3.

Co-culture experiments. As for the co-culture experiment, a transwell system was used $(0.4-\mu \mathrm{m}$ pore size semi-permeable membrane, BD Biosciences). In brief, $\mathrm{CD}_{4} 5^{+}$cells or $\mathrm{Lin}^{-} \mathrm{CD} 127^{+}$-depleted $\mathrm{CD} 45^{+}$cells $\left(5 \times 10^{5}\right.$ well $\left.^{-1}\right)$ were sorted from SVFs isolated from obese T2D subjects, cultured in the upper chamber of the transwell insert in a 12-well plate, and SVFs isolated from adipose tissue of control subjects $\left(1 \times 10^{6}\right.$ well $\left.^{-1}\right)$ were seeded in the lower chamber of the transwell insert. Meanwhile, trehalose-6,6'-dimycolate ( $5 \mu \mathrm{g}$ well ${ }^{-1}$, Nacalai Tesque, Kyoto, Japan) and palmitate $(200 \mu \mathrm{M}$, Sigma) were added in the lower chamber. After co-culture for $72 \mathrm{~h}, \mathrm{SVF}$ in the lower chamber were collected for further detection.

In another set of co-culture experiment, $2 \times 10^{8}$ cells of the SVF from obese T2D patients were magnetically enriched for ILCs using a negative selection kit that depletes non-ILCs, according to the manufacturer's protocol (EasySep ${ }^{\mathrm{TM}}$ Human ILCs Enrichment kit, STEMCELL Technologies). Then, adipose ILCs were cultured in the upper chamber of the transwell insert in a 12-well plate, and SVFs isolated from adipose tissue of control subjects $\left(1 \times 10^{6}\right.$ well $\left.^{-1}\right)$ were seeded in the lower chamber of the transwell insert. Meanwhile, trehalose-6,6'-dimycolate, palmitate, recombinant human IL-12 (R\&D Systems), and recombinant human IL-18 (R\&D Systems) were supplemented in the upper chamber, with neutralizing IFN- $\gamma$ antibody (BioXCell) or IgG isotype control antibody (BioXCell) added in separate group. SVFs isolated from adipose tissue of control subjects were cultured alone and determined as control group. After co-culture for $72 \mathrm{~h}$, SVFs in the lower chamber were collected for further detection.
As for the co-culture experiment using BMDMs and adipose ILC1s from C57BL/6 mice, detailed information were described on the Supplementary Methods section.

RNA extraction and quantitative PCR. Adipose tissues, which were snap-frozen in nitrogen gas and stored at $-80^{\circ} \mathrm{C}$, and SVFs were homogenized in TRIzol Reagent (15596026, Life Technologies, Thermo Fisher Scientific) and mixed with chloroform. After spinning down, the upper aqueous phase was mixed with the same volume of $70 \% \mathrm{EtOH}$ and applied to the RNeasy Mini Kit column according to the manufacturer's instructions for the RNeasy Mini Kit (74104, QIAGEN). Extracted RNA was converted to cDNA (RR036A, TAKARA) according to the manufacturer's instructions. Relative gene expression levels were analyzed by quantitative PCR on a Real-Time PCR system (LightCycler 480 II Roche, Switzerland). Fold differences in gene expression were calculated as $2^{-} \triangle \triangle \mathrm{Ct}$ using $\beta$-actin as the housekeeping gene. Primers used are listed in Supplementary Table 4.

Western blotting. For western blotting assays, adipose tissues were homogenized in lysis buffer ( $1 \%$ Triton X-100, $50 \mathrm{nM}$ Tris $\mathrm{HCl}, 150 \mathrm{mM} \mathrm{NaCl}, 5 \mathrm{mM}$ EDTA, $1 \mathrm{mM}$ PMSF) with protease cocktail (Roche) and phosphatase inhibitors (1 mM $\left.\mathrm{Na}_{3} \mathrm{Vo}_{4}, 10 \mathrm{mM} \mathrm{NaF}\right)$. Equal amounts of protein $(30 \mu \mathrm{g})$ were separated by gel electrophoresis, transferred onto a PVDF membrane (Millipore, Billerica, MA), and incubated with primary antibodies against phosphorylated Smad3 (ab52903, Abcam, 1:1000), and total Smad3 (ab40854, Abcam, 1:2000). GAPDH (ab8245, Abcam, 1:4000) was used as a loading control. The protein in the membrane was visualized using a Bio-Rad Clarity Western ECL Substrate (1705060, Bio-Rad). Uncropped western blot images can be found in the Source Data file.

Immunohistological analysis. Adipose tissue samples ( 200-300 mg) and the liver were washed with PBS, fixed in paraformaldehyde for $24 \mathrm{~h}$, paraffin embedded, and sliced into $4-\mu \mathrm{m}$ sections. The sections of adipose tissue were stained with Masson's trichrome dye or $0.1 \%$ Sirius Red in saturated picric acid for fibrosis assessment. The presence of F4/80-positive macrophages, pSTAT1, and aSMApositive area in VAT were detected. Immunohistochemistry was performed using the following antibodies: pSTAT1 (ab109461, Abcam, 1:50), F4/80 (ab6640 Abcam, 1:50), aSMA (ab7817, Abcam, 1:50). The sections of the liver were stained with hematoxylin and eosin. The quantitative histological analysis was performed by two investigators who had no knowledge of the origin of the slides. Staining images were captured and digitalized using an Olympus microscope (Olympus, Tokyo, Japan). Positive staining areas containing fibrillary collagens, as shown either with blue in Masson's trichrome stain or red in Sirius Red stain, were quantified by NIH Image J software.

Immunofluorescence. After deparaffinization and rehydration, adjacent 4- $\mu \mathrm{m}$ adipose tissue sections from mice were incubated with $10 \mathrm{mM}$ citric acid and heated in a microwave to recover antigenicity. Permeabilization was performed in PBS with Triton X-100 and nonspecific binding was blocked with $10 \%$ normal goat serum in PBS. Adipose tissue sections were incubated with F4/80 antibody (ab6640, Abcam, 1:50), TGF- $\beta 1$ antibody (ab92486, Abcam, 1:50), and pSTAT1 antibody (ab109461, Abcam, 1:50) in PBS with 3\% BSA and $0.1 \%$ Tween-20 at $4{ }^{\circ} \mathrm{C}$ overnight. Tissue sections were incubated for $1 \mathrm{~h}$ at room temperature with Alexa Fluor 488-conjugated AffinPure goat anti-rabbit IgG (GB25303, Servicebio, 1:50) and Cy3-conjugated goat anti-rabbit IgG (GB21303, Servicebio, 1:50). The cell nuclei were visualized by the mounting solution with DAPI (G1012, Servicebio). Staining images were captured and digitalized using a Nikon Eclipse 80i microscope.

Statistical analyses. All statistical analyses were performed using SPSS version 22.0 (SPSS Inc., USA). The data were presented as mean \pm SD or $n(\%)$. Differences in continuous variables in two groups were determined by the Student's $t$ test, and differences in categorical variables were determined by the $\chi^{2}$ analysis or Fisher exact test. Group differences were compared with ANOVA tests for normally distributed variables, whereas nonparametric Mann-Whitney $U$ test was performed for skewed parameters. Spearman's bivariate correlation tests and partial correlation analyses were conducted to study the associations. $P$-values $<0.05$ were considered statistically significant.

Reporting summary. Further information on research design is available in the Nature Research Reporting Summary linked to this article.

\section{Data availability}

Raw data for Figs. 1-6 and Supplementary Figs. 1 and 2 are presented in the Source Data file. All other data that support the findings of this study are available from the corresponding author upon reasonable request.

Received: 13 December 2018 Accepted: 4 July 2019

Published online: 22 July 2019 


\section{References}

1. Collaboration NCDRF. Trends in adult body-mass index in 200 countries from 1975 to 2014: a pooled analysis of 1698 population-based measurement studies with 19.2 million participants. Lancet 387, 1377-1396 (2016).

2. Dale, C. E. et al. Causal associations of adiposity and body fat distribution with coronary heart disease, stroke subtypes, and type 2 diabetes mellitus: a Mendelian randomization analysis. Circulation 135, 2373-2388 (2017).

3. Selvin, E., Parrinello, C. M., Sacks, D. B. \& Coresh, J. Trends in prevalence and control of diabetes in the United States, 1988-1994 and 1999-2010. Ann. Intern. Med. 160, 517-525 (2014).

4. Ji, L. et al. Primacy of the $3 \mathrm{~B}$ approach to control risk factors for cardiovascular disease in type 2 diabetes patients. Am. J. Med. 126, 925 e911-922 (2013).

5. McLaughlin, T., Ackerman, S. E., Shen, L. \& Engleman, E. Role of innate and adaptive immunity in obesity-associated metabolic disease. J. Clin. Investig. 127, 5-13 (2017).

6. Artis, D. \& Spits, H. The biology of innate lymphoid cells. Nature 517, 293-301 (2015).

7. Fuchs, A. ILC1s in tissue inflammation and infection. Front. Immunol. 7, 104 (2016).

8. Serafini, N., Vosshenrich, C. A. \& Di Santo, J. P. Transcriptional regulation of innate lymphoid cell fate. Nat. Rev. Immunol. 15, 415-428 (2015).

9. Bernink, J. H. et al. Human type 1 innate lymphoid cells accumulate in inflamed mucosal tissues. Nat. Immunol. 14, 221-229 (2013).

10. Bernink, J. H. et al. Interleukin-12 and -23 control plasticity of CD127(+) group 1 and group 3 innate lymphoid cells in the intestinal lamina propria. Immunity 43, 146-160 (2015).

11. O’Sullivan, T. E. et al. Adipose-resident group 1 innate lymphoid cells promote obesity-associated insulin resistance. Immunity 45, 428-441 (2016).

12. Lawler, H. M. et al. Adipose tissue hypoxia, inflammation, and fibrosis in obese insulin-sensitive and obese insulin-resistant subjects. J. Clin. Endocrinol. Metab. 101, 1422-1428 (2016).

13. Baker, N. A. et al. Diabetes-specific regulation of adipocyte metabolism by the adipose tissue extracellular matrix. J. Clin. Endocrinol. Metab. 102, 1032-1043 (2017).

14. Abdennour, M. et al. Association of adipose tissue and liver fibrosis with tissue stiffness in morbid obesity: links with diabetes and BMI loss after gastric bypass. J. Clin. Endocrinol. Metab. 99, 898-907 (2014).

15. Hasegawa, Y. et al. Repression of adipose tissue fibrosis through a PRDM16GTF2IRD1 complex improves systemic glucose homeostasis. Cell Metab. 27, 180-194 e186 (2018)

16. Keophiphath, M. et al. Macrophage-secreted factors promote a profibrotic phenotype in human preadipocytes. Mol. Endocrinol. 23, 11-24 (2009).

17. Tanaka, M. et al. Macrophage-inducible C-type lectin underlies obesityinduced adipose tissue fibrosis. Nat. Commun. 5, 4982 (2014).

18. Jang, J. E. et al. Nitric oxide produced by macrophages inhibits adipocyte differentiation and promotes profibrogenic responses in preadipocytes to induce adipose tissue fibrosis. Diabetes 65, 2516-2528 (2016).

19. Iwata, $\mathrm{H}$. et al. PARP9 and PARP14 cross-regulate macrophage activation via STAT1 ADP-ribosylation. Nat. Commun. 7, 12849 (2016).

20. Brestoff, J. R. \& Artis, D. Immune regulation of metabolic homeostasis in health and disease. Cell 161, 146-160 (2015).

21. Nishimura, S. et al. CD8+effector T cells contribute to macrophage recruitment and adipose tissue inflammation in obesity. Nat. Med. 15, 914-920 (2009).

22. Wensveen, F. M. et al. NK cells link obesity-induced adipose stress to inflammation and insulin resistance. Nat. Immunol. 16, 376-385 (2015).

23. Luo, T. et al. AMPK activation by metformin suppresses abnormal extracellular matrix remodeling in adipose tissue and ameliorates insulin resistance in obesity. Diabetes 65, 2295-2310 (2016).

24. Yadav, H. et al. Protection from obesity and diabetes by blockade of TGFbeta/Smad3 signaling. Cell Metab. 14, 67-79 (2011).

25. Wynn, T. A. \& Ramalingam, T. R. Mechanisms of fibrosis: therapeutic translation for fibrotic disease. Nat. Med. 18, 1028-1040 (2012).

26. Saini, R. \& Singh, S. Inducible nitric oxide synthase: an asset to neutrophils. J. Leukoc. Biol. 105, 49-61 (2019).

27. Lawless, S. J. et al. Glucose represses dendritic cell-induced T cell responses. Nat. Commun. 8, 15620 (2017)

28. Jianjun, Y. et al. T cell-derived inducible nitric oxide synthase switches off Th17 cell differentiation. J. Exp. Med. 210, 1447-1462 (2013).

29. Boulenouar, S. et al. Adipose type one innate lymphoid cells regulate macrophage homeostasis through targeted cytotoxicity. Immunity $\mathbf{4 6}$, 273-286 (2017).

30. American Diabetes A. 2. Classification and diagnosis of diabetes. Diabetes Care 40, S11-S24 (2017).
31. Xuan, Y. et al. Positive association between serum levels of bone resorption marker CTX and HbAlc in women with normal glucose tolerance. J. Clin Endocrinol. Metab. 100, 274-281 (2015).

32. Sondergaard, E., Espinosa De Ycaza, A. E., Morgan-Bathke, M. \& Jensen, M. D. How to measure adipose tissue insulin sensitivity. J. Clin. Endocrinol. Metab. 102, 1193-1199 (2017).

33. Gastaldelli, A. et al. Importance of changes in adipose tissue insulin resistance to histological response during thiazolidinedione treatment of patients with nonalcoholic steatohepatitis. Hepatology 50, 1087-1093 (2009).

34. Trim, W., Turner, J. E. \& Thompson, D. Parallels in immunometabolic adipose tissue dysfunction with ageing and obesity. Front. Immunol. 9, 169 (2018).

\section{Acknowledgements}

This work was supported by the National Natural Science Foundation of China Grant Awards (81770819, 81570737, 81370947, 81570736, 81500612, 81400832, 81600637, 81600632 , and 81703294), the National Key Research and Development Program of China (2016YFC1304804 and 2017YFC1309605), the Jiangsu Provincial Key Medical Discipline (ZDXKB2016012), the Key Project of Nanjing Clinical Medical Science, Jiangsu Province Key Research and Development Program (BE2016606), the Jiangsu Provincial Medical Talent (ZDRCA2016062), the Natural Science Foundation of Jiangsu Province of China (BK20170125), the Scientific Research Foundation of "Project 333" in Jiangsu Province (GYHT20190013), the Jiangsu Provincial Medical Youth Talent (QNRC2016020, QNRC2016019, and QNRC2016018), the Medical Scientific Research Foundation of Jiangsu Province of China (Z201610 and Q2017006), the Science and Technology Project of Administration of Traditional Chinese Medicine of Jiangsu Province of China (YB2015072), the Six Talent Peaks Project of Jiangsu Province of China (WSN-165 and SWYY-091), the Fundamental Research Funds for the Central Universities (021414380444, 021414380092, 021414380208, 021414380160, 021414380142, 021414380279, 021414380296, and 021414380317), the Nanjing Science and Technology Development Project (ZKX16036, YKK16105, and 201605019), and the Nanjing Health Youth Talent (QRX17123)

\section{Author contributions}

H.D.W., L.S. and F.C.L. conceived the study, designed and performed in vitro experiments, analyzed and interpreted data. H.D.W. and F.C.L. performed statistical analysis and wrote the paper. W.H.F., X.T.S., C.P.J., X.H.C., P.Z.Z., C.J. and Y.W. conducted surgery and collected samples from patients. F.C.L. and X.Y. performed histological and mRNA expression analysis. H.D.W. performed co-culture experiments and animal studies. M.W.Z. supervised the project and interpreted the results. D.L.Z. supervised the project. Y.B. supervised the project, designed experiments, interpreted the results, and cowrote the paper. All authors had the opportunity to comment on the paper.

\section{Additional information}

Supplementary Information accompanies this paper at https://doi.org/10.1038/s41467 019-11270-1.

Competing interests: The authors declare no competing interests.

Reprints and permission information is available online at http://npg.nature.com/ reprintsandpermissions/

Peer review information: Nature Communications thanks Daniela Carlos and Kevin Hart for their contribution to the peer review of this work

Publisher's note: Springer Nature remains neutral with regard to jurisdictional claims in published maps and institutional affiliations.

Open Access This article is licensed under a Creative Common Attribution 4.0 International License, which permits use, sharing, adaptation, distribution and reproduction in any medium or format, as long as you give appropriate credit to the original author(s) and the source, provide a link to the Creative Commons license, and indicate if changes were made. The images or other third party material in this article are included in the article's Creative Commons license, unless indicated otherwise in a credit line to the material. If material is not included in the article's Creative Commons license and your intended use is not permitted by statutory regulation or exceeds the permitted use, you will need to obtain permission directly from the copyright holder. To view a copy of this license, visit http://creativecommons.org/ licenses/by/4.0/

(C) The Author(s) 2019 